\title{
A theoretical study of Maker Fringe measurements in poled multi-layer silica structures focusing on the impact of layer quantity and spacing
}

by

Pouyan Nasr

B.Eng. (Carleton) 2012

A thesis submitted to the Faculty of Graduate and Postdoctoral Affairs in partial fulfillment of the requirements for the degree of

Master of Applied Science

in

Electrical and Computer Engineering

Ottawa-Carleton Institute for Electrical and Computer Engineering Department of Electronics

Carleton University

Ottawa, Ontario

December 2015

(C) 2015, Pouyan Nasr 


\section{Abstract}

A second order nonlinearity in silica glass, which does not exist intrinsically, can be achieved through thermal poling. The poling process breaks the intrinsic symmetry of the silica allowing for even ordered non-linearities. After poling was first successfully demonstrated in 1991, the achievable nonlinearity has remained weak (after several attempts). Recently, some progress has been made through the development of multi-layer silica structures created through alternating dopant concentration. Larger observed nonlinearities in these samples (through the creation of second harmonic radiation) suggest that this may have created a distributed non-linearity that could result in more efficient frequency doubled laser sources. The impact of layer spacing on the observed nonlinearity has remained an open question. In this thesis a numerical model is developed with the goal investigating the impact of the quantity and spacing between layers on second harmonic generation. The thesis begins with a brief history on poling techniques and various attempts to induce greater second order nonlinearities in silica. We then develop the theoretical model that describes second harmonic generation in multi-layer structures. This section is followed by a description of our experiment which basically is simulation model and the thesis finishes with a discussion of the conclusions drawn from the model. 


\section{Acknowledgements}

Firstly, I would like to express my sincere gratitude to my advisor Professor Christopher Smelser for the continuous support of my master study and related research, for his patience, motivation, and immense knowledge. His guidance helped me throughout the research and writing of this thesis. I could not have imagined having a better advisor and mentor for my Masters study. His example inspired me to improve myself as a researcher and as a person, and will continue to do so in the future.

Besides my advisor, I would like to thank my fellow lab-mates and office-mates for the stimulating discussions, for the sleepless nights we were working together before deadlines, and for all the fun we have had in the last two years. I am also grateful to Mr. Nagui Mikhail, facility manager, who helped me a lot with my office and computer equipment.

Last but not least, I would like to thank my family, my parents, specifically my dear sister who helped me tremendously in my life generally and particularly with writing and formatting this thesis, my grandmother and uncle for their unconditional love and spiritual support throughout writing this thesis and my life in general. 


\section{Contents}

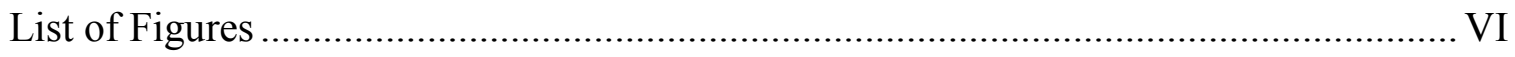

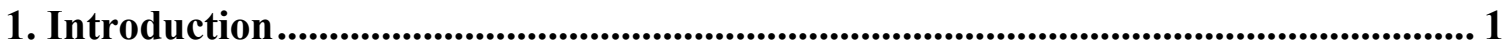

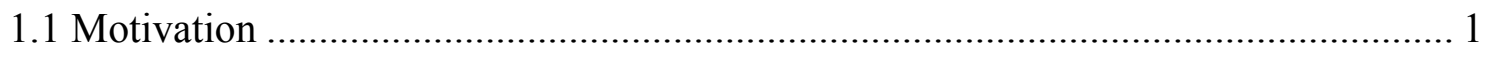

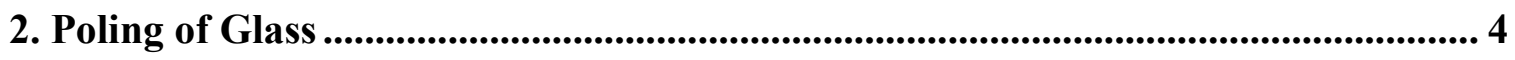

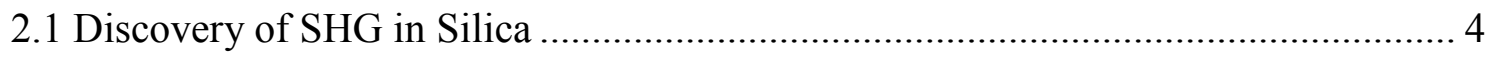

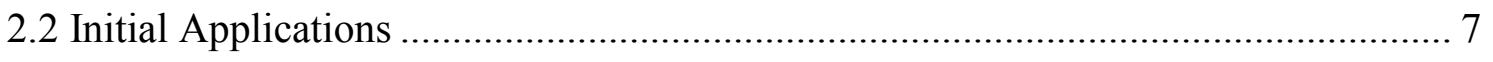

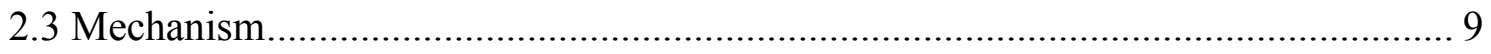

2.4 Multi-layer structures; A Breakthrough ..................................................... 12

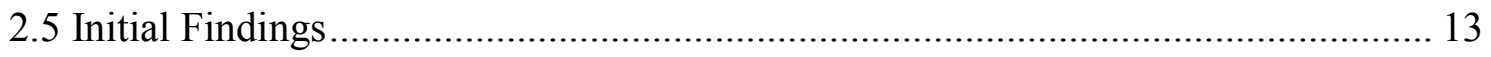

3. Second Harmonic Generation: Theory and Model................................................ 19

3.1 Time-Independent SHG coupled mode equations ............................................ 19

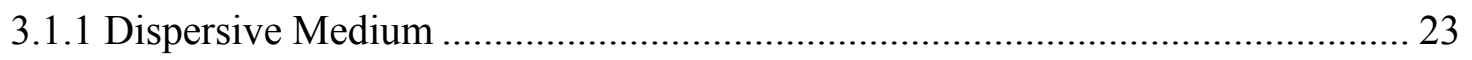

3.2 The Nonlinear Polarization Term ...................................................................... 25

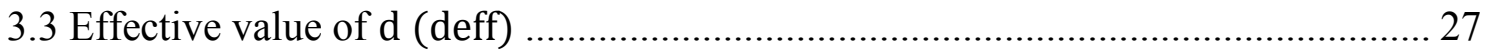

3.4 Coupled-wave equations for sum-frequency and SHG .................................... 34

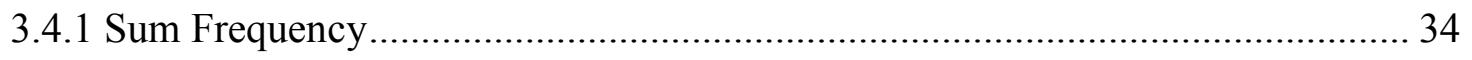

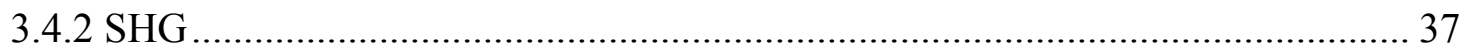

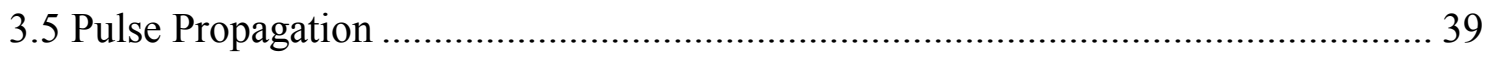

3.6 Time-Dependent SHG Coupled Mode Equations ............................................. 43

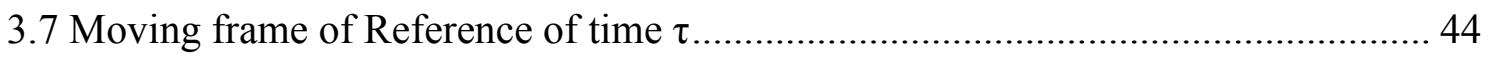

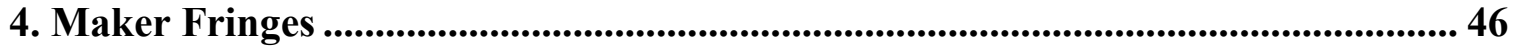

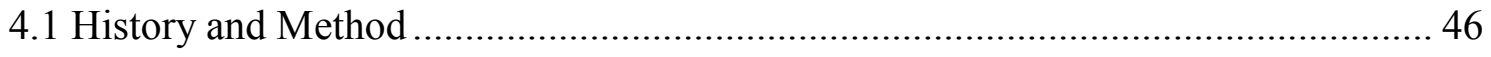




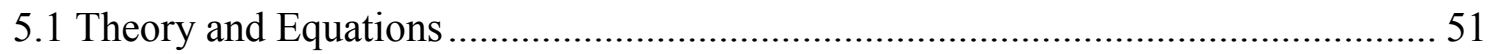

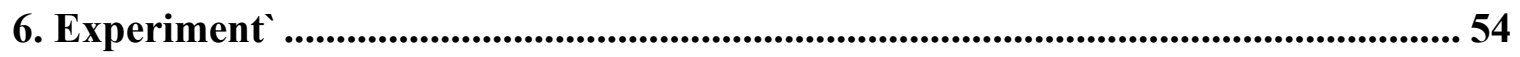

6.1 Femtosecond Maker Fringes: time domain study …...................................... 54

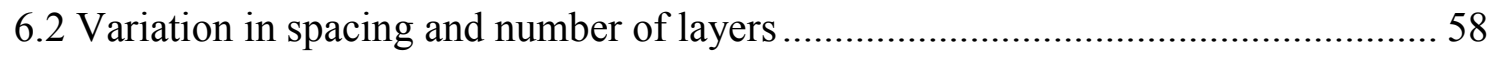

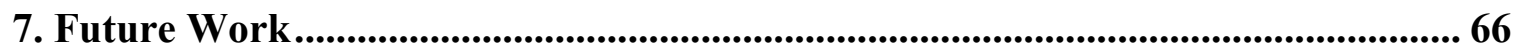

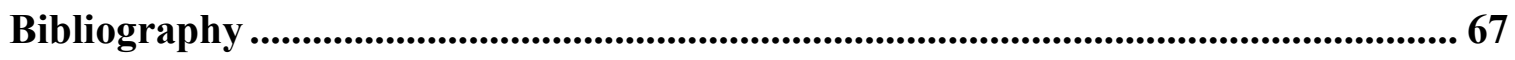

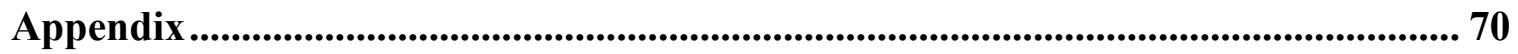

Matlab Code for the Model .................................................................................. 70 


\section{List of Figures}

2.1 Experimental setup to illuminate the sample with Nd:Yag laser source .................... 5

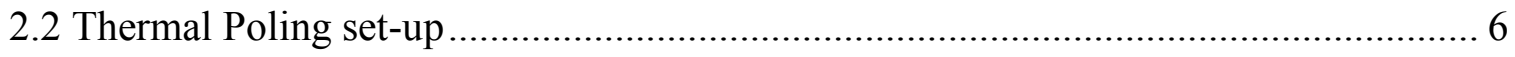

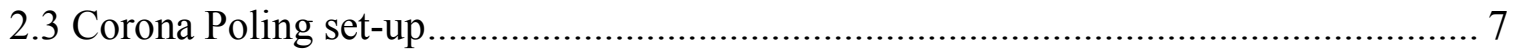

2.4 Mach-Zehnder Interferometer set-up ........................................................ 8

2.5 Near-field second-harmonic patterns in thermally poled fused silica with a periodic

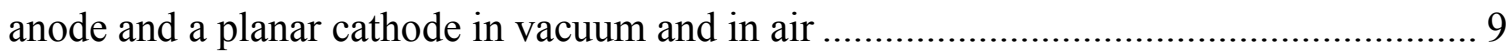

2.6 The Two Mechanisms believed to be occurring during thermal poling ................... 10

2.7 Depletion layer is formed near the anode surface ................................................. 10

2.8 Overlap of the multilayer structure with the waveguide mode ............................... 13

2.9 Structures investigated in [13]. P and B doped layered structures were poled and

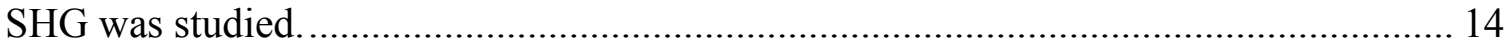

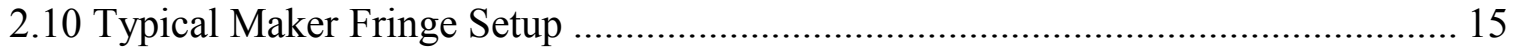

2.11 Maker Fringe pattern for poled multi-layer structure depicted in Fig. 8. V.S.

Theoretical Maker Fringe pattern for the sample.................................................... 16

2.12 Two-layer structure consisting of 1 micron thick Ge-doped layers separated by undoped silica. (b) Nano-layer structure consisting of 40 segments of Ge-doped/undoped

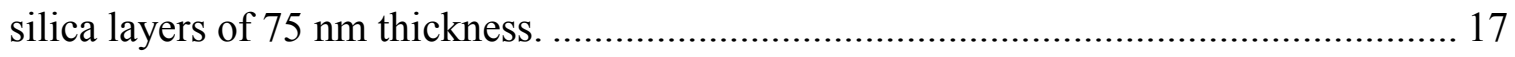

2.13 SHG in thermally poled bulk silica glass and the silica-based multilayered structures

3.1 Principal plane of the crystal (kZ) and: (a) Ordinary beam. (b) Extraordinary beam. 31

3.2 Sample being rotated in Maker Fringe method................................................... 33

3.3 Sum Frequency Generation and Second Harmonic Generation .............................. 35 
4.1 Propagation of the fundamental and the second harmonic waves during a Maker

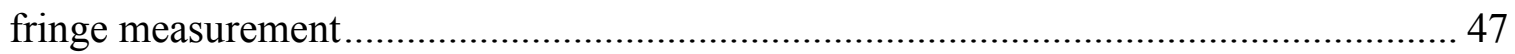

5.1 Two schemes of pulse propagation in nonlinear dispersive media............................. 53

6.1 The multi-layer stacks were on either side of the substrate ......................................... 54

6.2 Theoretical Maker Fringe pattern for the sample in Fig. \# considering a long picosecond length fundamental pulse .......................................................................... 55

6.3 Maker Fringe pattern for the sample in Fig. 6.1 considering a long picosecond length

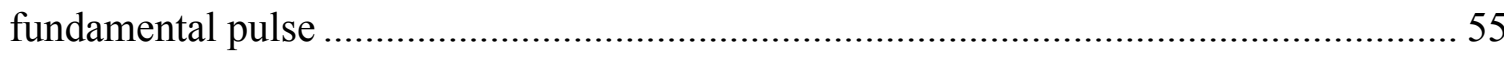

6.4 Modeled Maker Fringe pattern for the sample in Fig. (6.1) considering an 80

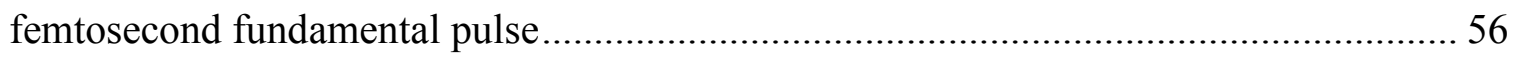

6.5 The mechanism for the reduction in fringe visibility with fs fundamental pulse lengths

6.6 Second Harmonic light generated at either side of the sample. Clearly, there is very

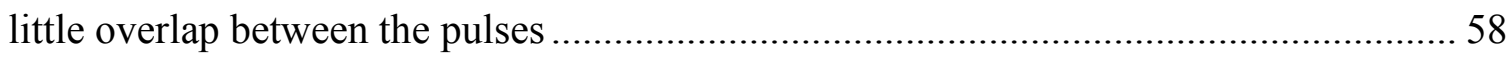

6.7 The two samples with equal thickness but different separation................................... 59

6.8 Three structures of our model. (a) 40-layer structure. (b) Stretched 40 layer structure (Separation increasing). (c) Stretched structure with constant length (separation increasing, number of layers reducing consequently)

6.10 SHG of sample (b) normalized to SHG of (a). We see that the SHG dramatically decreases with layer spacing

6.11 Ratio of SHG generated by the 40 layered structure (a) to that generated with (c) from Fig. 6.9 


\section{Chapter 1}

\section{Introduction}

\section{Motivation}

The field of photonics encompasses a wide variety of applications in areas such as communications, health, structural monitoring and bio-chemical sensing, amongst others, and has an impact on our lives virtually every minute of every day. The most heralded of all photonics inventions, and what really transformed the optics field into the industry that it is currently, was the invention of the laser. Lasers, of course, come in a wide variety of shapes and sizes and are used in ways as varied as laser welding and cutting, long-haul telecommunications, surgery, or even fundamental studies of molecular dynamics.

With an impact comparable to that of the laser, shortly after its invention came the development of the first low loss silica telecommunications fiber. Since the bandwidth that is supported in telecommunications fiber is several hundred $\mathrm{THz}$ it is possible to transmit large amounts of information through the fiber. In the ensuing years the fiber-based telecommunications field exploded resulting in an enormous infrastructure for the development of inexpensive silica fiber based optical components. Silica has proven over the years to be a very fortunate choice of material for its many, many desirable properties. Some of these properties would include: low loss, immunity to electromagnetic 
interference, large window of transparency, low thermal expansion coefficient, sensitivity to UV radiation when doped with germanium, and its ability to be formed into a wide variety of compact devices [1].

On account of its material benefits and its relative abundance there has been a significant research effort aimed at the development of silica based fiber laser sources. The motivation behind this effort stems from more than the use of inexpensive materials as fiber lasers are also much more compact, reliable and robust than their free space counterparts. On account of the efforts in the research community the output power of fiber lasers has increased over 2 orders of magnitude in the last 10 years alone. Free space fiber lasers are composed of many components, from mirrors, polarizers, gain materials, to frequency doublers. The vast majority of the components of free space lasers have been duplicated with fiber based technologies. This means that much of the functionality of free space lasers is also present in the fiber based versions. There are still some drawbacks to fiber lasers, such as the low damage threshold for many of the components that limit the peak power, however, research is constantly being conducted to try to mitigate these limitations.

One component that has not yet been duplicated using a silica based component is the frequency doubler. Frequency doublers are non-linear optical components that are commonly used to generate visible radiation from lasers operating in the near infrared (which are half the frequency of visible radiation) region of the spectrum. The process of frequency doubling is also often referred to as second harmonic generation (SHG). In free space lasers frequency doublers are commonly made out of materials that have a high second order non-linearity ( $\mathrm{SON})$. One such material, lithium niobate, is very commonly used for frequency doubling and light modulation [2]. The use of non-linear materials like 
lithium niobate with fiber laser designs is non-ideal as it cannot be easily incorporated into the fiber design and must be placed external to the laser cavity. The extra-cavity configuration can introduce additional loss on account of Fresnel reflections from the surface of lithium niobate and the doubling efficiency could also be affected due to mismatch in the thermal expansion coefficients of the fiber laser components. It is obvious, on account of these limitations that the ideal frequency doubling component would be silica based as it could easily be incorporated into the laser design with no Fresnel reflection or thermal mismatch.

The main drawback of silica for use as a non-linear optical component for frequency doubling applications stems from the fact that silica is an amorphous material [2]. An amporphous, or centrosymmetric material is forbidden from having an even ordered non-linear susceptibility. As the frequency doubled radiation generated from a doubling crystal is related to its $2^{\text {nd }}$ order optical non-linear susceptibility, also denoted as $\chi^{(2)}$, silica cannot be used to generate frequency doubled radiation [3]. It seems then that it is physically impossible for a silica based optical component to be used to replace a nonlinear crystal in the laser design. 


\section{Chapter 2}

\section{Poling of Glass}

\subsection{Discovery of SHG in Silica}

Lithium niobate is a non-centrosymmetric crystalline material that possesses a very large second order non-linearity on the order of $81 \mathrm{pm} / \mathrm{V}$ [4]. It is the ideal material for second harmonic generation. As mentioned above, the centro-symmentric nature of silica makes it impossible for it to be used in the same capacity, even if it would be ideal from a compatibility standpoint.

This picture began to change after the startling discovery of SHG emanating from silica optical fiber during a 1986 study by Margulis et al [5]. Margulis coupled the output of a pulsed Nd:YAG laser into a Ge-doped fiber. Initially they did not observe any green light (which is the second harmonic of the Nd:YAG laser), however, after a period of steady illumination a weak second harmonic was detected. After this stage the intensity of the green light at the output grew exponentially. The induced SON using optical poling was quite weak $\left(\chi^{2} \sim 10^{-3} \mathrm{pm} / \mathrm{V}\right)$. The rate of growth was found to be largely dependent on the IR optical power. The green intensity saturated and stopped growing after several hours (about 12 hours). Over short periods of time they observed that the SON would remain 
constant. If the sample was left for several days there would be a reduction in the SON, however, this was recoverable with continued exposure.

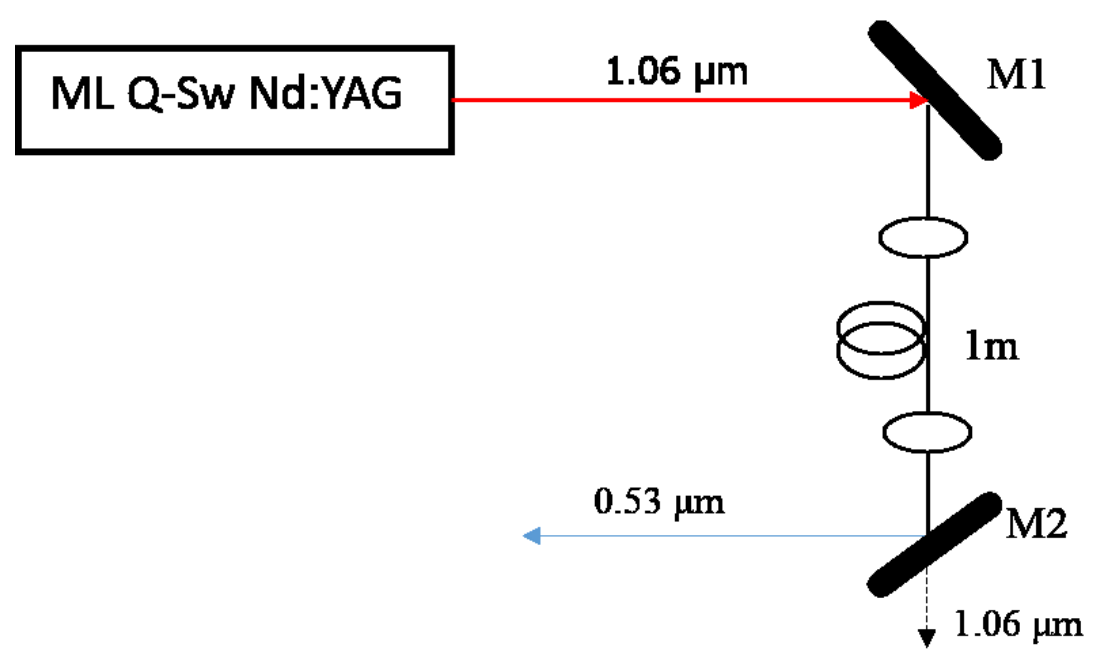

Figure 2.1. Experimental setup to illuminate the sample with Nd:YAG Laser source. ML, mode locked

The discovery of SHG in silica based optical fiber was obviously of great interest to the research community. It was still not known why the material became non-linear but it was certainly believed that the strong electric field somehow changed the composition of the material to allow for second harmonic generation.

The observation of SHG in silica fiber convinced other researchers to find ways to obtain even higher intensities. Eventually in 1991, another significant advancement in inducing a second order nonlinearity in silica glass was observed, called thermal poling [6]. Thermal poling is a process, shown in Fig.2, where a glass sample is heated to several hundred degrees celcius while subjected to a strong electric field (by applying a dc voltage of 5-6 kV across the sample). It was discovered that, after the poling process, a permanent 
SON was induced in the glass sample. In this study the obtained SON had a susceptibility $\chi^{(2)}=1 \mathrm{pm} / \mathrm{V}$ which, compared to existing crystals like lithium niobate that have susceptibilities of $81 \mathrm{pm} / \mathrm{V}$, is relatively small. Even though the resulting magnitude was small, practical devices may have been realizable given a large enough interaction length between the incident light and the non-linearity. By etching the sample with an HF acid solution the induced non-linearity was determined to exist in a thin region near the surface (less than $4 \mu \mathrm{m}$ close to anode).

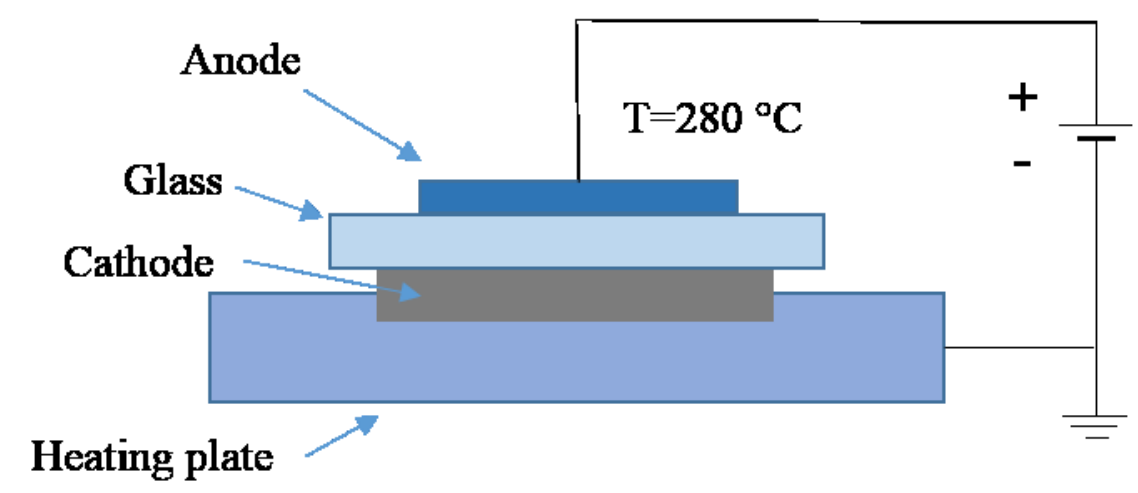

Figure 2.2. Thermal poling set-up

An alternate poling method, called corona poling, was used to create the first phasematched SHG from a glass waveguide in 1992 [8]. In the corona poling method an electric field is applied to the glass through a needle-to-plane electrode geometry, shown in Fig.3. A tungsten needle was placed about $1 \mathrm{~cm}$ above the grounded planar aluminum electrode and a voltage of $5 \mathrm{kV}$ was applied to the needle at both 100 and $300{ }^{\circ} \mathrm{C}$. With this method it was estimated that the induced $\mathrm{SON}$ in the material reached $0.5 \mathrm{pm} / \mathrm{V}$. 


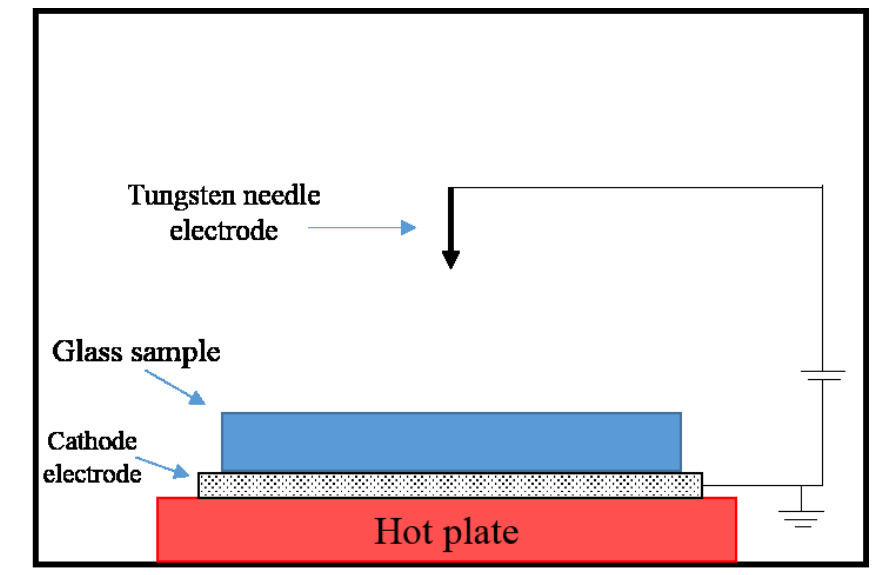

Figure 2.3. Corona poling set-up

Following the thermal and corona poling experiments other methods were tested as well which resulted in almost the same magnitude of SON as previously observed. These methods included: CO2 laser-assisted poling [9], UV poling [10], UV-fs poling [11], electron-beam poling [12], and proton implantation poling [13].

\subsection{Initial Applications}

The first demonstration of electro-optic phase modulation in fused silica was reported in 1993 where the nonlinearity was induced by thermal poling in an electron-beam-irradiated waveguide [13].

In that experiment a waveguide was fabricated by electron-beam irradiation of fused-silica substrate with a low-energy electron beam and was subsequently thermally poled. The sample was then placed in an arm of a Mach-Zehnder interferometer. For the wavelength of $\lambda=633 \mathrm{~nm}$ and device interaction length of $4.8 \mathrm{~mm}$ the observed phase shift in the poled phase modulator was measured to be $32 \mathrm{mrad}$ when the applied electric field was 7.3 
$\mathrm{V} / \mu \mathrm{m}$. The phase shift was found to depend linearly on the applied voltage up to the maximum voltage of $1100 \mathrm{~V}$. It was believed that this low phase shift is the result of poor overlap $(\sim 2 \%)$ between the nonlinear region and the waveguide mode.

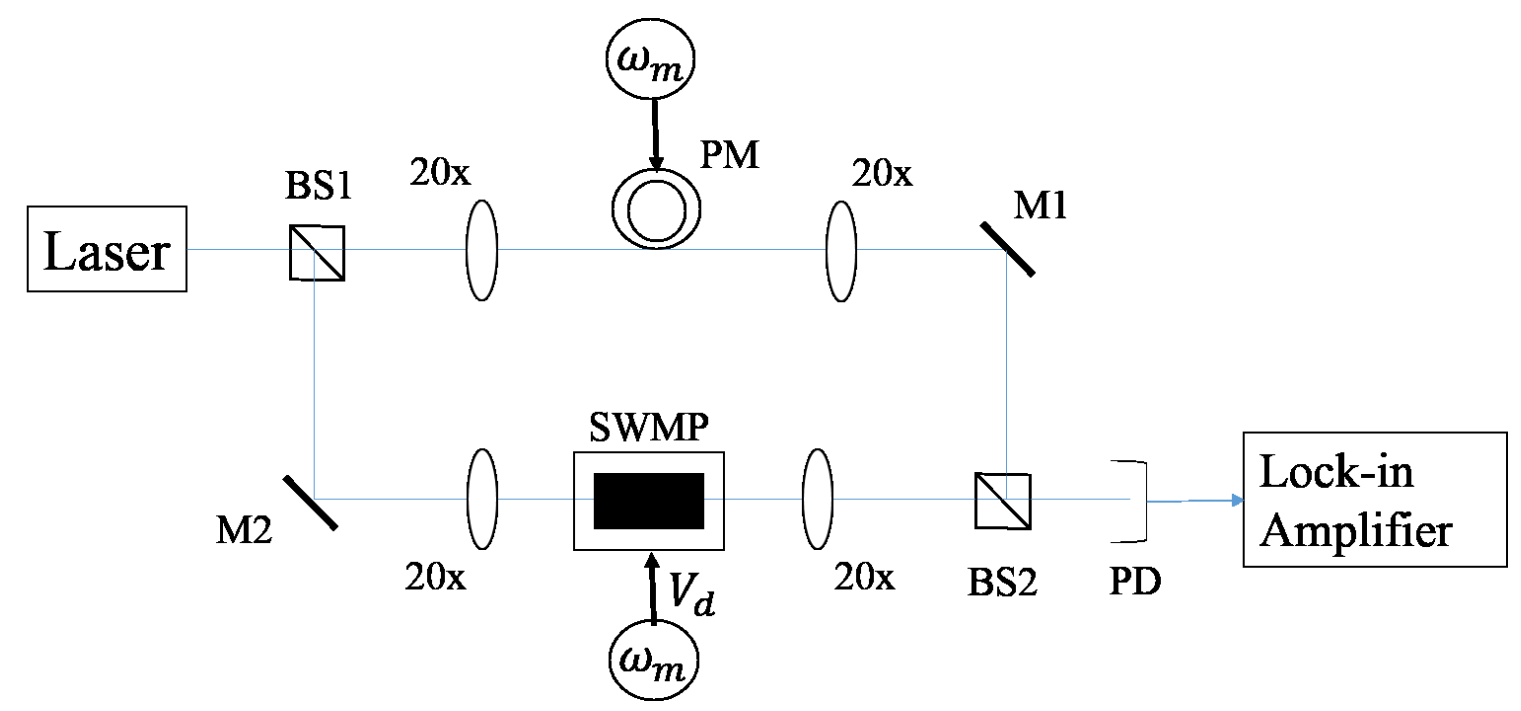

Figure 2.4. Mach-Zehnder interferometer setup: SWPM (silica waveguide phase modulator); PM (fiber piezoelectric phase modulator) BS's (beam splitters) M's (mirrors); PD (photodetector); 20x's (20x's magnification lenses).

In 1994 an experiment conducted by Kazansky reported frequency doubling and generation of blue light in a periodically patterned quasi-phase-matched optical fiber. The bandwidth was measured to be $0.78 \mathrm{~nm}$ which is close to theoretical calculations.

To achieve that configuration they used a periodic thermal poling technique on fused silica in vacuum. Periodic poling lead to quasi-phase matching of the fundamental and doubled light which increased the SHG efficiency. The contrast for poling was significantly improved when it was performed in vacuum. 

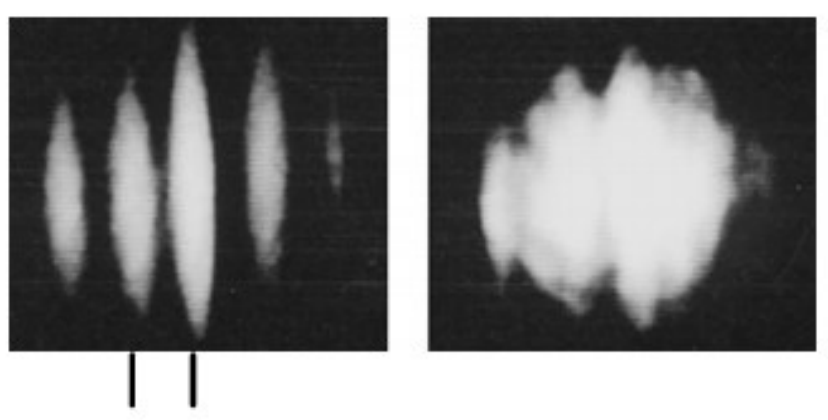

\section{$20 \mu \mathbf{m}$}

Figure 2.5. Near-field second-harmonic patterns in thermally poled fused silica with a periodic anode and a planar cathode in vacuum (left-hand side) and with air (right-hand side). The greatly improved contrast for poling in vacuum is noticeable.[14]

\subsection{Mechanism}

It was now possible to produce an artificial second order non-linearity in silica, however, it was still not known what mechanism was responsible for the inducement of the nonlinearity. Essentially, two models were proposed as front runners to explain the inducement of the second order non-linearity. The first proposed model claimed that the non-linearity is caused by the migration of charged ions within the glass during the poling process which leads to a permanent electric field within the glass [15]. While the glass is heated ions can migrate through the glass toward the cathode but, when the glass is cooled, the carriers are trapped causing a permanent separation of charges resulting in the establishment of an internal electric field. This field would then combine with the intrinsic third order non-linearity of the material (more on this later) to produce an effective second order non-linearity.

The second model that was proposed suggested the orientation of molecules within the glass with the external electric field at high temperatures [16]. This realignment would 
then break the symmetry in the glass, allowing for non-zero second order nonlinearities to exist in the glass. A comparison of the two models is shown in the Figure below.

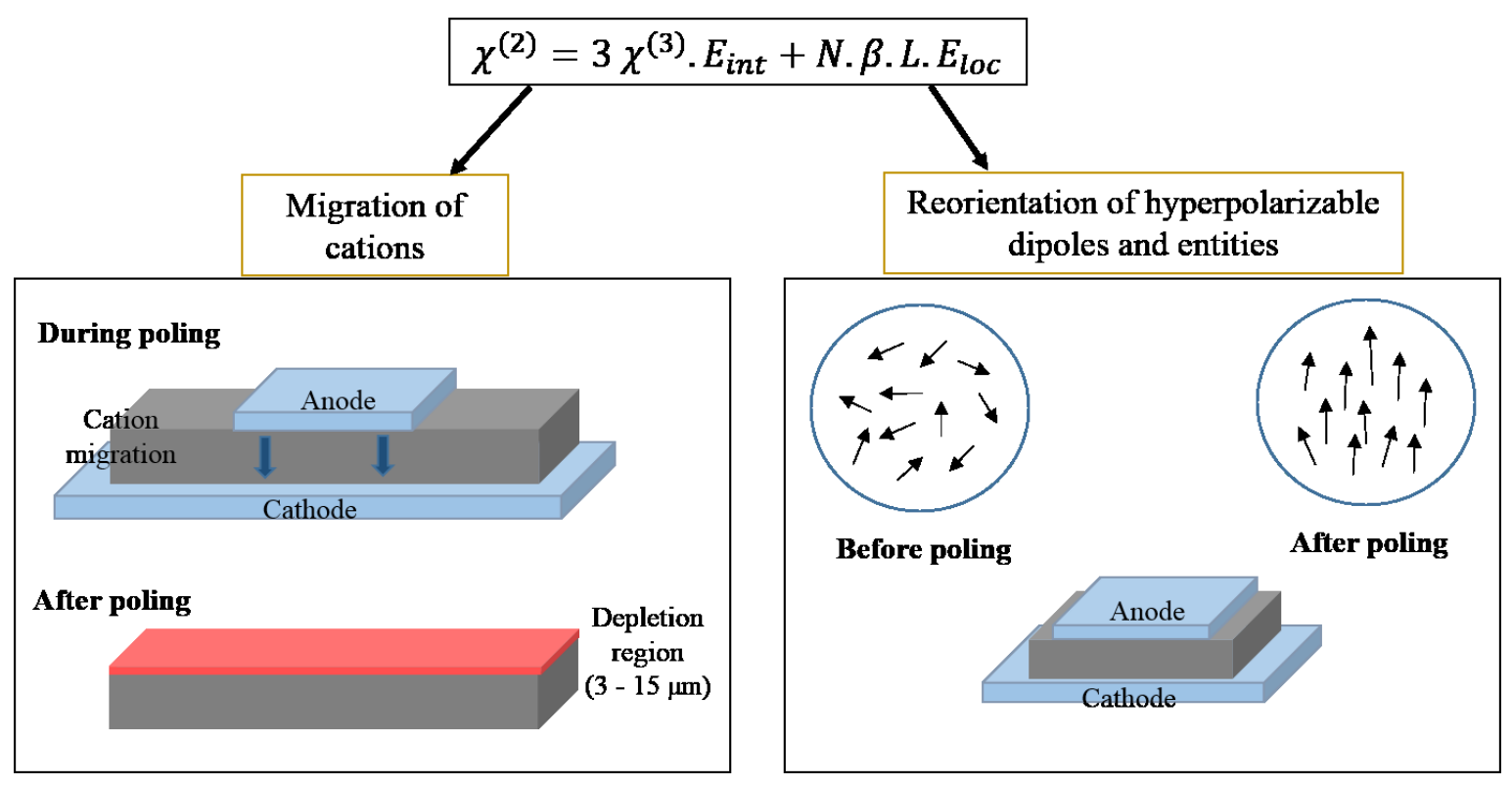

Figure 2.6. The Two mechanism believed to be occurring during thermal poling

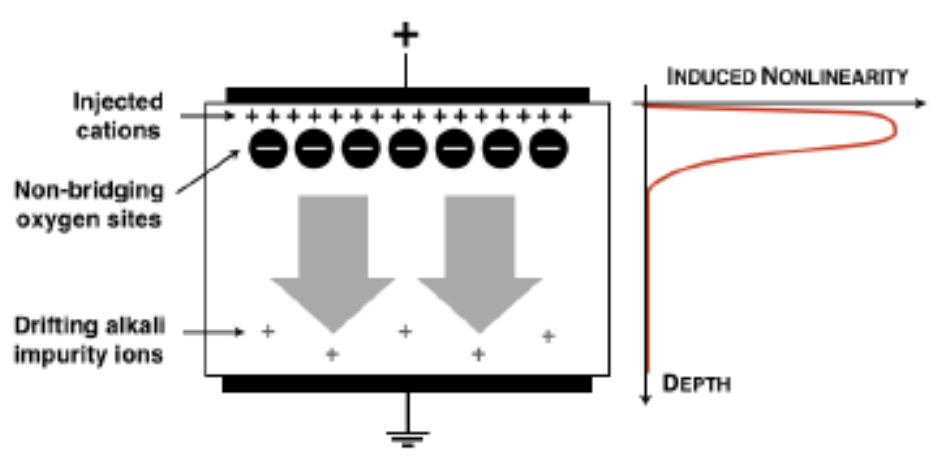

Figure 2.7. Depletion layer is formed near the anode surface [26]

Over time it has generally become clear that the first mechanism is likely responsible for the observed SON [17]. Positive charged alkali ions (such as $\mathrm{Na}+$ and $\mathrm{Li}+$ ), which normally exist in glass, are bound to negatively charged non-bridging oxygen sites. In the presence of the high voltage and temperature the ions would have enough mobility to move 
away from the anode site and migrate toward the cathode resulting in a negatively charged depletion region. A positively charged layer is also formed at the anodic surface. Studies that looked at the impact of the atmosphere in poling showed that when the anode electrode is not in contact with the glass, local ionization produces a layer consisting of hydrogenated ions (e.g. $\mathrm{H}+$ or $\mathrm{H} 3 \mathrm{O}+$ ), which negate the depletion region. However, when the anode electrode is in contact with the glass, prohibiting exposure to the atmosphere, formation of these ions is prohibited [18]. A strong electric field is formed because of the charge potential difference between the positive-charge layer and negative-charge depletion region. When the heater is turned off and temperature of the sample goes down to room temperature, the ions will freeze in place, even after switching the voltage off.

Several researchers have studied the time evolution of the SON in glasses and concluded that it is likely that there are multiple time periods of charge migration that impact the induced electric field $[19,20]$. These researchers have shown that there is an initial fast charge migration that is responsible for the inducement of the SON within the first few minutes when the field is applied to the sample, then slow migration would occur which causes long term changes in the SON profile.

After about a decade of intensive research and without significant improvement (the highest achieved magnitude of SON was only $1.6 \mathrm{pm} / \mathrm{V}$ [2] compared to $\sim 81 \mathrm{pm} / \mathrm{V}$ for lithium niobate) on the magnitude of the SON coefficient, interest on this research area waned. The other main issue, the small size of the non-linear region and the resulting small overlap and effective SON, was still a significant hurdle to implementing these devices. One solution to the overlap problem was increasing the interaction length, however, this presented other obstacles to using these new devices in optical modulators. Either 
researchers had to find a way to significantly increase the induced non-linearity or think of how to improve the overlap of the non-linearity and the fundamental mode of a waveguide. As we'll see in the next section the second approach is starting to show some promise.

\section{$2.4 \quad$ Multi-layer structures: A Breakthrough}

According to previous experimental measurements on thin films the induced SON, and thus the active layer of SHG, only exists in a very thin layer of glass near the anode. As researchers continued to study poling of glass in various configurations it became apparent that there was also an accumulation of SON near the interfaces between regions of disparate dopant concentration or composition. Several studies reported the peaking of SHG at the interface between different types of glass [21, 22]. Research also showed that the interface between pure silica glass and doped silica could also effectively introduce a spike in the SON $[23,24]$. It was believed that the doped silica prevented the migration of ions through the material, causing the buildup of the SON at an interface [c]. This, initially, could be regarded as undesirable as this would prohibit the creation of a non-linearity across a waveguide (for example a Ge-doped silica guide). The blocking of ions at the interface of a waveguide and its cladding is likely responsible for the low phase shift observed in experiments incorporating the poled silica in modulators [13].

While the lowered mobility of charges in doped layers could be viewed as an impediment it is also possible to use it to one's advantage to produce a device that has heightened overlap of the SON with the fundamental mode of the waveguide. If a multilayer structure was produced containing alternating doped and un-doped layers it may be 
possible to create a larger non-linear region that overlaps well with the effective mode of either an imbedded waveguide or a fiber structure, as shown in Fig. 2.8. The overall nonlinearity produced in each layer would be same as that produced with only one layer, so this does not solve that problem, but the stack would create a much stronger overlap with the fundamental mode of the guide and should make any non-linear interaction much more efficient.
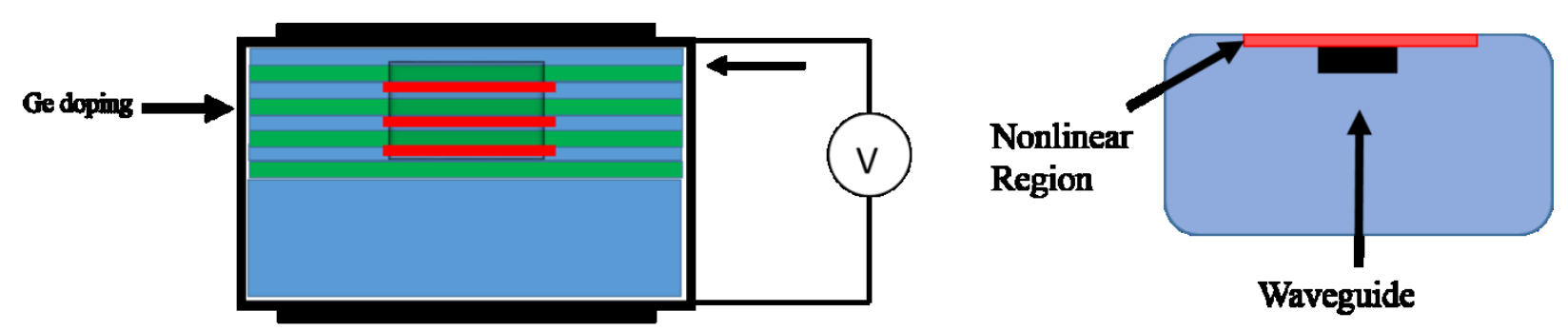

Figure 2.8. Overlap of the multilayer structure with the waveguide mode

\subsection{Initial Findings}

The use of multi-layer structures to increase the penetration depth of the SON into a sample was the subject of a previous study by a former student at Carleton University (Ksenia Yadav). This study involved two main experiments [25, 26].

In the first experiment, which was designed to test whether a non-linearity could be produced on either side of a sample, P-doped multilayered silica-based structures were deposited by low-pressure chemical vapor deposition (LPCVD) at $420{ }^{\circ} \mathrm{C}$ on double-side polished synthetic fused substrates (ES grade by Tosoh Quartz). The initial structure that was investigated is shown below in Fig. 2.9. 


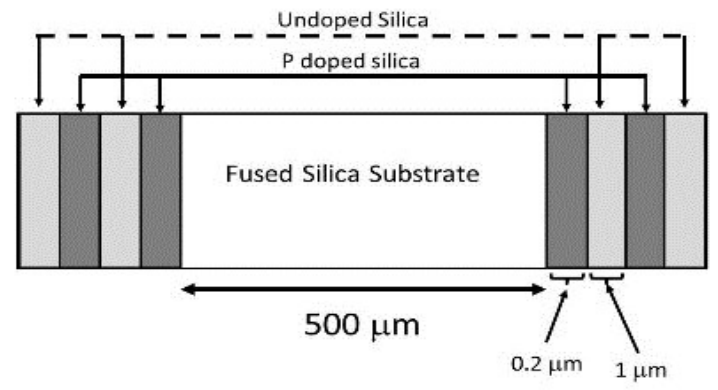

Figure 2.9. Structures investigated in [13]. P and B doped layered structures were poled and SHG was studied.

These samples were then poled using a corona poling setup at $300{ }^{\circ} \mathrm{C}$ for 8 minutes in a nitrogen filled chamber with a poling voltage of $6000-9000 \mathrm{~V}$. Following the poling the samples were allowed to cool to room temperature before the poling voltage was switched off.

After these samples were poled the induced second order non-linearity in the material could be analyzed by measuring the samples ability to produce second harmonic radiation through a Maker Fringe measurement shown in Fig. 2.10. These measurements were conducted with ultrafast mode-locked Ti:sapphire laser (operating wavelength $800 \mathrm{~nm}$, pulse width $<70 \mathrm{fsec}$, repetition rate $80 \mathrm{MHz}$ ). The p-polarized beam was passed through the sample as it was being rotated in the plane of incidence (Z-k plane) from $-80^{\circ}$ to $80^{\circ}$, relative to the incident laser beam. Second harmonic light was then generated in the sample. This light is captured by a photomultiplier tube after a bandpass filter. As the sample rotates the path length through the material increases. The generated SHG will cycle from a maximum to a minimum for each increase in length equivalent to the coherence length. This can occur several times, resulting in a fringe pattern. In nonlinear optics, the coherence length is normally known as the length over which fundamental and 
harmonic wave get out of phase with the phase difference of $\pi$ or 180 degrees (in another convention it is the phase difference of $2 \pi$ ).

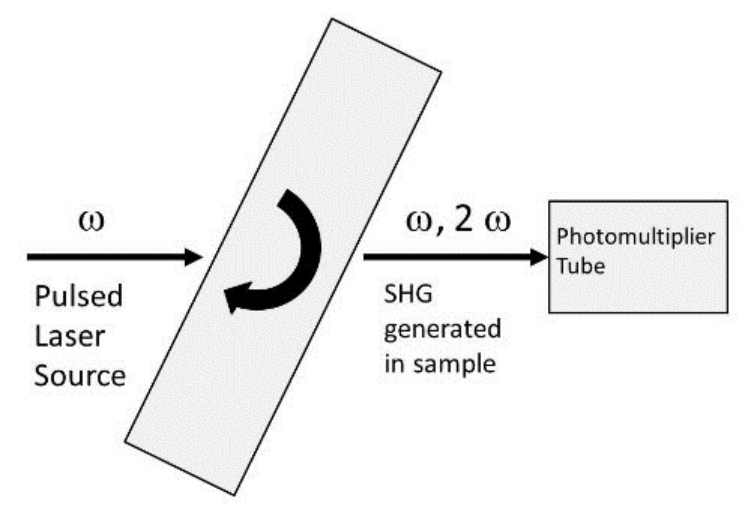

Figure 2.10. Typical Maker Fringe Setup. A pulsed laser is passed through a non-linear sample and SHG is detected with a photo-multiplier tube.

The resulting Maker Fringe measurement for the sample shown in Fig. 2.9 is shown in Fig. 2.11 (a). We see that on top of a background envelope there are a number of small fringes. This indicates that a non-linear region was successfully induced on either side of the sample. In Fig. 2.11 (b) we show what the expected theoretical Maker Fringe pattern should look like for this configuration given that an equal non-linearity was induced on either side of the sample. It is evident that the measured fringes and the theory do not match. One possible explanation for this observation was that the non-linearity on one side of the sample was significantly stronger than the other. HF etching experiments were performed on this sample which indicated that the non-linearity induced on either side of the sample was equivalent. The explanation for the reduced fringe visibility was left as an open question [25]. 


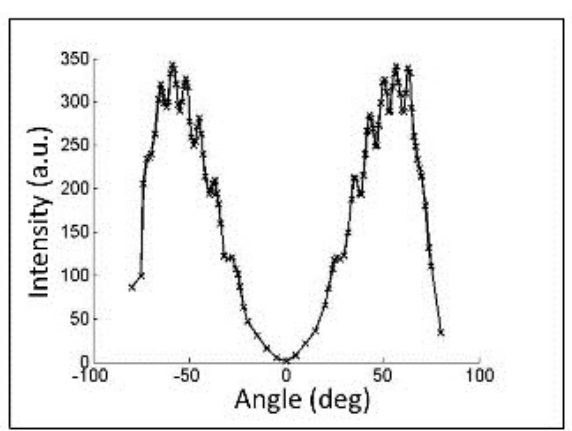

(a)

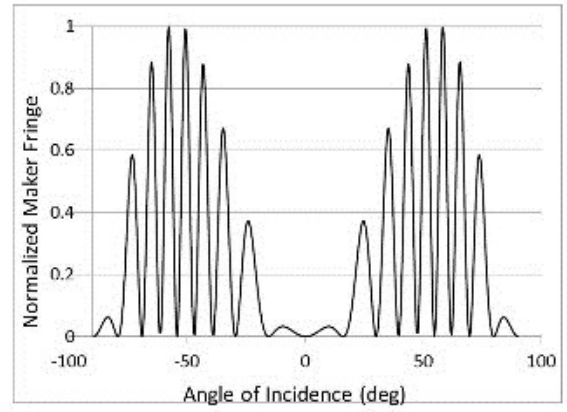

(b)

Figure 2.11. (a) Maker Fringe pattern for poled multi-layer structure depicted in Fig. 8. (b) Theoretical Maker Fringe pattern for the sample.

In a second study, that sought to compare the SHG from a few layers to a many layer structure, single sided samples were created using a plasma enhanced chemical vapor deposition (PECVD) apparatus [26]. Two basic configurations were compared. The first configuration, shown in Fig. 2.12 (a), was a structure with 2 Ge-doped layers separated by 1 micron and a total thickness of $3 \mu \mathrm{m}$. The second sample, depicted in Fig. 2.12 (b), was comprised of 40 layers separated by $75 \mathrm{~nm}$ for a total thickness also equal to $3 \mu \mathrm{m}$. These samples were compared to a pure silica sample without layers.

These samples were poled using a contact poling technique where the sample was placed between two n-type electrodes and heated to $300{ }^{\circ} \mathrm{C}$ at $3000 \mathrm{~V}$. After 8 minutes the heat was switched off and the sample was allowed to cool for 1.5 hours, at which point the voltage was switched off. 


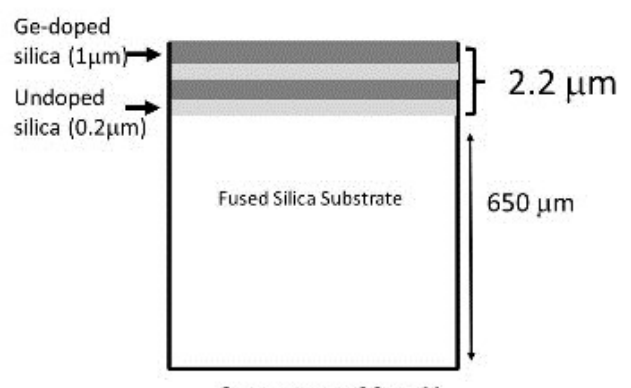

Ge content: 22 wt $\%$

(a)

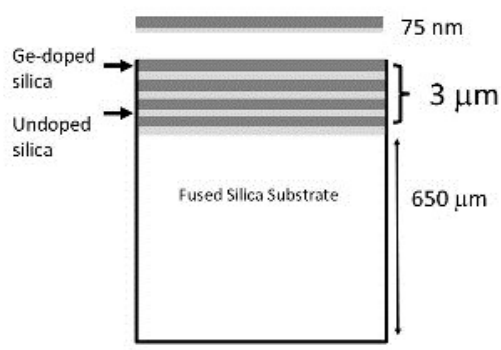

Ge content: 0.2 wt $\%$

(b)

Figure 2.12. (a) Two-layer structure consisting of 1 micron thick Ge-doped layers separated by undoped silica. (b) Nano-layer structure consisting of 40 segments of Ge-doped/undoped silca layers of $75 \mathrm{~nm}$ thickness.

These samples were then measured with the same Maker Fringe method described above. The second harmonic produced from the 40-layer structure proved to be 204 times that produced in a bulk silica sample as shown in Fig.12. (B-bulk silica, G-2 layer, M-40 layer), and approximately 80 times that produced in the 2 layer structure.

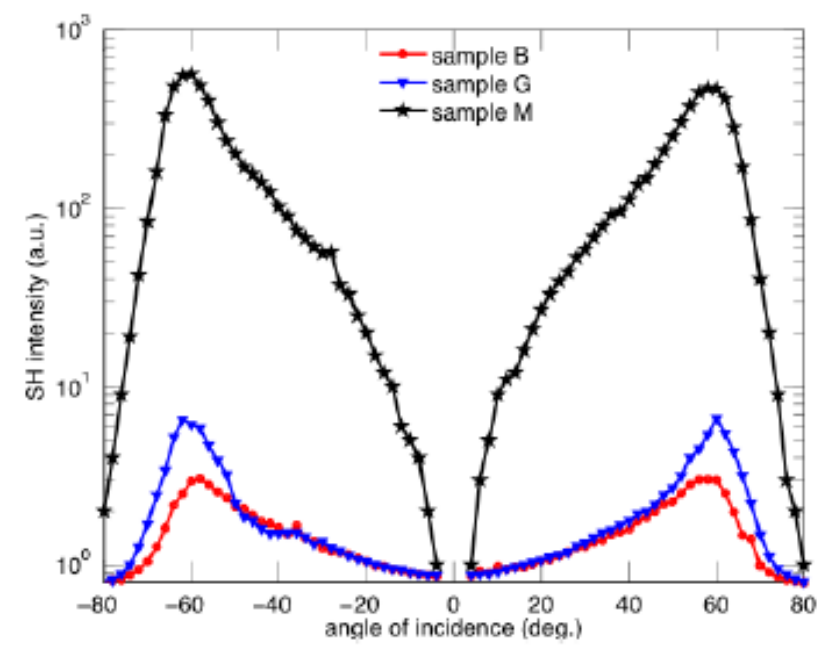

Figure 2.13. SHG in thermally poled bulk silica glass and the silica-based multilayered structures. A two-fold SHG enhancement is obtained in sample $G$ compared to sample $B$, where the two samples were poled under identical conditions. A 204-fold enhancement is obtained in sample $M$, which consisted of a 3- $\mu$ m-thick stack with a large number of sub-100 nm-thick nanolayers. 
If the increase in the observed SHG was a consequence of an increase in $\chi^{(2)}$, which is not likely, this would represent a 14-fold increase in the non-linearity over that of fused silica. The reason for the increase in SHG is likely a result of the inducement of multiple nonlinear regions within the sample. The impact of the separation between the layers on the SHG measurement was not considered in this study.

Germanium dopant substitutes for $\mathrm{Si}^{4+}$ when it is doped into silica glass. Germanium has equal numbers of valence electrons as silicon therefore it does not change the charge of the lattice. The improved nonlinearity in the multilayered samples was because the region at the interfaces between the germanium-doped and undoped silica act as barriers against the migration of the positive charges. These barriers act in two ways to enhance the nonlinearity. Firstly, the accumulation of impurity alkali ions close to the interfaces may create strong localized nonlinearity peaks. Secondly, the barriers could prevent the detrimental positive ions (which are injected from the anode) to move further into the sample [18]. From the first study it is clear that phosphorous doped silica can also be used as a blocking layer [25].

The discovery that a multi-layer stack could significantly increase the SHG from a poled silica sample has the potential to be a major breakthrough in the field of poled silica. There were a couple of open questions, however, that were not fully explored in the above mentioned studies. First, the mechanism for the reduction in the visibility of the Maker fringes was not fully explored. Second, the impact that the layer spacing could have on the generated SHG was not considered as well. The remainder of this thesis develops the model that was used to examine these open questions, along with the findings of the study. 


\section{Chapter 3}

\section{Second Harmonic Generation: Theory and Model}

In the following sections we will develop the model that was used to simulate the Maker fringe experiments that were conducted by Yadav [25, 26]. We will also use this model to explore some of the implications of the spacing between and number of layers on SHG in poled multi-layer silica structures. We will begin with Maxwell's equations.

\subsection{Time-Independent SHG coupled mode equations}

From Maxwell's equations [3]:

$$
\begin{aligned}
& \nabla \cdot \mathbf{D}_{(\mathbf{r}, t)}=\rho_{(\mathbf{r}, t)} \\
& \nabla \cdot \mathbf{B}_{(\mathbf{r}, t)}=0
\end{aligned}
$$

(Faraday' s law)

$$
\nabla \times \mathbf{E}_{(\mathbf{r}, t)}=-\frac{\partial \mathbf{B}_{(\mathbf{r}, t)}}{\partial t}
$$

(Ampere's law)

$$
\nabla \times \mathbf{H}_{(\mathbf{r}, t)}=\mathbf{J}_{(\mathbf{r}, t)}+\frac{\partial \mathbf{D}_{(\mathrm{r}, t)}}{\partial t}
$$


Where $\rho_{(\mathbf{r}, t)}$ is the density of free charges, and $\mathbf{J}_{(\mathbf{r}, t)}$ is the surface charge density current. B and $\mathrm{D}$ are formulated as follow:

$$
\begin{gathered}
\mathbf{D}_{(\mathbf{r}, t)}=\varepsilon_{0} \mathbf{E}_{(\mathbf{r}, t)}+\mathbf{P}_{(\mathbf{r}, t)} \\
\mathbf{B}_{(\mathbf{r}, t)}=\mu_{0}\left[\mathbf{H}_{(\mathbf{r}, t)}+\mathrm{M}_{(\mathbf{r}, t)}\right]
\end{gathered}
$$

where $\mathbf{P}_{(\mathrm{r}, t)}$ is the electric polarization or macroscopic polarization density (electric dipole moment per unit volume), and $\mathbf{M}_{(\mathbf{r}, t)}$ is the magnetic polarization or magnetization (magnetic dipole moment per unit volume) of the medium.

There are two main frequent assumptions in nonlinear optics; primarily there are no free charges in the regions that equations are being applied (hence no charge density), secondly no magnetic polarization is present. By these two assumptions we can write;

$$
\begin{array}{ll}
\text { 1. } \rho_{(\mathbf{r}, t)}=0, & \mathbf{J}_{(\mathbf{r}, t)}=0 \\
\text { 2. } \mathbf{M}_{(\mathbf{r}, t)}=0 &
\end{array}
$$

The nonlinearity in the material could be represented through the relation of $\mathbf{D}$ and $\mathbf{E}$ as:

$$
\mathbf{D}_{(\mathbf{r}, t)}=\varepsilon_{0} \mathbf{E}_{(\mathbf{r}, t)}+\mathbf{P}_{(\mathbf{r}, t)}
$$

Where polarization vector $\mathbf{P}$ has nonlinear dependence on electric field $\mathbf{E}$.

Now the optical wave equation can be derived in the normal method from Faraday's law. 


$$
\begin{aligned}
\nabla \times \nabla \times \mathbf{E}_{(\mathbf{r}, t)} & =-\frac{\partial}{\partial t} \nabla \times \mathbf{B}_{(\mathbf{r}, t)} \\
& =-\mu_{0} \frac{\partial}{\partial t} \nabla \times \mathbf{H}_{(\mathbf{r}, t)} \\
& =-\mu_{0} \frac{\partial}{\partial t}\left(\frac{\partial \mathbf{D}_{(\mathbf{r}, t)}}{\partial t}\right)=-\mu_{0} \frac{\partial^{2} \mathbf{D}_{(\mathbf{r}, t)}}{\partial t^{2}}
\end{aligned}
$$

now replace D by Eq. (3.8) to obtain:

$$
\nabla \times \nabla \times \mathbf{E}_{(\mathbf{r}, t)}=-\mu_{0}\left(\varepsilon_{0} \frac{\partial^{2} \mathbf{E}_{(\mathbf{r}, t)}}{\partial t^{2}}+\frac{\partial^{2} \mathbf{P}_{(\mathbf{r}, t)}}{\partial t^{2}}\right)
$$

By knowing that speed of light in vacuum $=\frac{1}{\sqrt{\mu_{0} \varepsilon_{0}}}$, the above equation can be modified to obtain the most general form of the wave equation in nonlinear optics:

$$
\nabla \times \nabla \times \mathbf{E}_{(\mathbf{r}, t)}+\frac{1}{C^{2}} \frac{\partial^{2} \mathbf{E}_{(\mathbf{r}, t)}}{\partial t^{2}}=-\mu_{0} \frac{\partial^{2} \mathbf{P}_{(\mathbf{r}, t)}}{\partial t^{2}}
$$

By using the identity vector equations, the first term of the left hand side would be represented as

$$
\nabla \times \nabla \times \overrightarrow{\mathbf{E}}_{(\mathbf{t})}=\nabla\left(\nabla \cdot \mathbf{E}_{(\mathbf{t})}\right)-\nabla^{2} \mathbf{E}_{(\mathbf{t})}
$$

In the linear optics of isotropic source-free media since there are no free charges, $\rho=0$, therefore $\nabla \cdot \mathbf{D}=0$, meaning that $\nabla \cdot \mathbf{E}=0$, thus the first term on the right hand side of the above equation can be dropped. Therefore the wave equation would reduce to 


$$
\nabla^{2} \mathbf{E}-\frac{1}{c^{2}} \frac{\partial^{2} \mathbf{E}_{(\mathbf{r}, t)}}{\partial t^{2}}=\frac{1}{\varepsilon_{0} c^{2}} \frac{\partial^{2} \mathbf{P}_{(\mathbf{r}, t)}}{\partial t^{2}}
$$

Since $\mathbf{D}_{(\mathbf{r}, t)}=\varepsilon_{0} \mathbf{E}_{(\mathbf{r}, t)}+\mathbf{P}_{(\mathbf{r}, t)}$ the above equation can be expressed as

$$
\nabla^{2} \mathbf{E}-\frac{1}{\varepsilon_{0} c^{2}} \frac{\partial^{2}}{\partial t^{2}} \mathbf{D}_{(\mathbf{r}, t)}=0
$$

Polarization $\mathbf{P}$ is composed of linear part $\mathbf{P}^{(1)}$, which is linearly dependant on the electric field $\mathbf{E}$, and nonlinear $\mathbf{P}^{N L}$ part

$$
\mathbf{P}=\mathbf{P}^{(1)}+\mathbf{P}^{N L}
$$

Similarly the displacement field $\mathbf{D}$ can be decomposed into its linear and nonlinear components as

$$
\mathbf{D}=\mathbf{D}^{(1)}+\mathbf{P}^{N L}
$$

Where the linear part $\mathbf{D}^{(1)}$ is described as

$$
\mathbf{D}^{(1)}=\varepsilon_{0} \mathbf{E}+\mathbf{P}^{(1)}
$$

By substituting all of these the wave equation can be stated as

$$
\nabla^{2} \mathbf{E}-\frac{1}{\varepsilon_{0} c^{2}} \frac{\partial^{2}}{\partial t^{2}} \mathbf{D}^{(1)}=\frac{1}{\varepsilon_{0} c^{2}} \frac{\partial^{2}}{\partial t^{2}} \mathbf{P}^{N L}
$$


This form of the wave equation is useful for the case of a lossless and dispersion-less medium. In such a medium $\mathbf{D}$ would be expressed as

$$
\mathbf{D}^{(1)}=\varepsilon_{0} \varepsilon^{(1)} . \mathbf{E}
$$

Where $\varepsilon^{(1)}$ is a dielectric tensor which is frequency-independent, dimensionless and relative permittivity that differs for each material. The wave equation for the case of isotropic and dispersion-less material becomes

$$
-\nabla^{2} \mathbf{E}+\frac{\varepsilon^{(1)}}{c^{2}} \frac{\partial^{2}}{\partial t^{2}} \mathbf{E}=-\frac{1}{\varepsilon_{0} c^{2}} \frac{\partial^{2}}{\partial t^{2}} \mathbf{P}^{N L}
$$

On this equation the right-hand side term is the nonlinear response of the medium which acts as a source term.

\subsubsection{Dispersive Medium}

In dispersive medium each field is represented as the summation of all its frequency components.

$$
\begin{aligned}
& \mathbf{E}_{(\mathbf{r}, t)}=\sum_{n} \mathbf{E}_{n(\mathbf{r}, t)} \\
& \mathbf{D}_{(\mathbf{r}, t)}^{(1)}=\sum_{n} \mathbf{D}_{n(\mathbf{r}, t)}^{(1)}
\end{aligned}
$$




$$
\mathbf{P}_{(\mathbf{r}, t)}^{N L}=\sum_{n} \mathbf{P}_{n(\mathbf{r}, t)}^{N L}
$$

Each frequency component on above equations can be expressed in terms of its complex amplitude as

$$
\begin{aligned}
& \mathbf{E}_{n(\mathbf{r}, t)}=\mathbf{E}_{n(\mathbf{r})} e^{-i \omega t}+c . c . \\
& \mathbf{D}_{n(\mathbf{r}, t)}^{(1)}=\mathbf{D}_{n(\mathbf{r})}^{(1)} e^{-i \omega t}+c . c . \\
& \mathbf{P}_{n(\mathbf{r}, t)}^{N L}=\mathbf{P}_{n(\mathbf{r})}^{N L} e^{-i \omega t}+\text { c.c. }
\end{aligned}
$$

In a dispersive medium since permittivity is a dielectric tensor which is variable with frequency, the relation between $\mathbf{D}$ and $\mathbf{E}$ can be shown as

$$
\mathbf{D}_{n(\mathbf{r}, t)}^{(1)}=\varepsilon_{0} \varepsilon_{\left(\omega_{n}\right)}^{(1)} . \mathbf{E}_{n(\mathbf{r}, t)}
$$

Now our wave equation can be written to be valid for each frequency component

$$
\nabla^{2} \mathbf{E}_{n}-\frac{\varepsilon_{\left(\omega_{n}\right)}^{(1)}}{c^{2}} \frac{\partial^{2}}{\partial t^{2}} \mathbf{E}=\frac{1}{\varepsilon_{0} c^{2}} \frac{\partial^{2}}{\partial t^{2}} \mathbf{P}_{n}^{N L}
$$




\subsection{The non-linear polarization term}

In linear optics, the induced polarization has linear and direct dependence on the electric field strength, as the product of susceptibility and electric field

$$
P_{(t)}=\varepsilon_{0} \chi^{(1)} E_{(t)}
$$

Where $\chi^{(1)}$ is called linear susceptibility

In nonlinear optics, the general form of the equation relating electric field to polarization is

$$
\begin{aligned}
P_{(t)} & =\varepsilon_{0}\left[\chi^{(1)} E_{(t)}+\chi^{(2)} E_{(t)}^{2}+\chi^{(3)} E_{(t)}^{3}+\cdots\right] \\
& \equiv P_{(t)}^{(1)}+P_{(t)}^{(2)}+P_{(t)}^{(3)}+\cdots
\end{aligned}
$$

$\chi^{(2)}$ and $\chi^{(3)}$ are recognised as second and third order nonlinear optical susceptibilities respectively. $\chi^{(1)}$ is a second rank tensor and $\chi^{(2)}$ is a third rank tensor and so on. In this thesis we will be primarily concerned with second order non-linear processes, specifically that of sum frequency generation and the special case of second harmonic generation. In the case of sum frequency generation the non-linear polarization term on the right hand side of equation (28) can be expressed in the following form,

$$
\begin{aligned}
\boldsymbol{P}_{i\left(\omega_{3}\right)}= & \varepsilon_{0} \sum_{j k}\left[\chi_{i j k\left(\omega_{3} ; \omega_{1}, \omega_{2}\right)}^{(2)} E_{j\left(\omega_{1}\right)} E_{k\left(\omega_{2}\right)}+\right. \\
& \left.\chi_{i j k\left(\omega_{3} ; \omega_{2}, \omega_{1}\right)}^{(2)} E_{j\left(\omega_{2}\right)} E_{k\left(\omega_{1}\right)}\right]
\end{aligned}
$$


Here $\omega_{3}=\omega_{1}+\omega_{2}$ satisfies conservation of energy [3]. If we assume the medium in which the field is propagating through is intrinsically symmetric then the nonlinear susceptibility tensor would possess permutation symmetry in its last two indices $j$ and $k$ that allow them to be interchangeable.

$$
\chi_{i j k\left(\omega_{n}+\omega_{m} ; \omega_{m}, \omega_{n}\right)}^{(2)}=\chi_{i j k\left(\omega_{n}+\omega_{m} ; \omega_{n}, \omega_{m}\right)}^{(2)}
$$

Hence

$$
\boldsymbol{P}_{i\left(\omega_{3}\right)}=2 \varepsilon_{0} \sum_{j k} \chi_{i j k\left(\omega_{3} ; \omega_{1}, \omega_{2}\right)}^{(2)} E_{j\left(\omega_{1}\right)} E_{k\left(\omega_{2}\right)}
$$

If the input fields are polarized and aligned along the same direction (for example $\mathrm{x}$ ), then

$$
\boldsymbol{P}_{i\left(\omega_{3}\right)}=2 \varepsilon_{0} \chi_{i x x\left(\omega_{3} ; \omega_{1}, \omega_{2}\right)}^{(2)} E_{x\left(\omega_{1}\right)} E_{x\left(\omega_{2}\right)}
$$

For the case of second harmonic generation where $\omega_{1}=\omega_{2}$ and hence $\omega_{3}=2 \omega_{1}$, the polarization would become

$$
\boldsymbol{P}_{i\left(\omega_{3}\right)}=\varepsilon_{0} \chi_{i x x\left(\omega_{3} ; \omega_{1}, \omega_{1}\right)}^{(2)} E_{x\left(\omega_{1}\right)}^{2}
$$




\subsection{Effective value of $d\left(d_{e f f}\right)$}

We now introduce a commonly used variation on the susceptibility,

$$
d_{i j k}=\frac{1}{2} \chi_{i j k}^{(2)}
$$

The coefficient fraction, $\frac{1}{2}$ exists due to historical convention. By substituting into the nonlinear polarization

$$
P_{i\left(\omega_{n}+\omega_{m}\right)}=\varepsilon_{0} \sum_{j k} \sum_{(n m)} 2 d_{i j k} E_{j\left(\omega_{n}\right)} E_{k\left(\omega_{m}\right)}
$$

When the Kleinman's symmetry condition is valid, which is when the incident and generated waves have frequencies much smaller than the lowest resonance frequency of the material, the susceptibility tensor $\chi_{i j k}$ is symmetric in its last two indices. This leads to $d_{i j k}$ also being symmetric in its last two indices. This is always valid for the case of second harmonic generation because $\omega_{n}$ and $\omega_{m}$ are identical and equal. Therefore the notations of the elements can be reduced to:

$$
d_{i j k}=d_{i l}
$$

Where

$$
\begin{array}{ccccccc}
j k: & 11 & 22 & 33 & 23,32 & 31,13 & 12,21 \\
l: & 1 & 2 & 3 & 4 & 5 & 6
\end{array}
$$


Hence the non-linear susceptibility tensor can be reduced to a $3 \times 6$ matrix instead of $3 \times 9$ matrix, consequently number of elements become 18 from 27

$$
d_{i l}=\left[\begin{array}{llllll}
d_{11} & d_{12} & d_{13} & d_{14} & d_{15} & d_{16} \\
d_{21} & d_{22} & d_{23} & d_{24} & d_{25} & d_{26} \\
d_{31} & d_{32} & d_{33} & d_{34} & d_{35} & d_{36}
\end{array}\right]
$$

The equation for the polarization of the sum-frequency generation in terms of susceptibility tensor $d_{i l}$ and electric field where $\omega_{3}=\omega_{1}+\omega_{2}$ hence is given by

$$
\begin{aligned}
& {\left[\begin{array}{ll}
P_{x\left(\omega_{3}\right)} \\
P_{y\left(\omega_{3}\right)} \\
P_{z\left(\omega_{3}\right)}
\end{array}\right]} \\
& =4 \varepsilon_{0}\left[\begin{array}{llllll}
d_{11} & d_{12} & d_{13} & d_{14} & d_{15} & d_{16} \\
d_{21} & d_{22} & d_{23} & d_{24} & d_{25} & d_{26} \\
d_{31} & d_{32} & d_{33} & d_{34} & d_{35} & d_{36}
\end{array}\right]\left[\begin{array}{c}
E_{x\left(\omega_{1}\right)} E_{x\left(\omega_{2}\right)} \\
E_{y\left(\omega_{1}\right)} E_{y\left(\omega_{2}\right)} \\
E_{z\left(\omega_{1}\right)} E_{z\left(\omega_{2}\right)} \\
E_{y\left(\omega_{1}\right)} E_{z\left(\omega_{2}\right)}+E_{z\left(\omega_{1}\right)} E_{y\left(\omega_{2}\right)} \\
E_{x\left(\omega_{1}\right)} E_{z\left(\omega_{2}\right)}+E_{z\left(\omega_{1}\right)} E_{x\left(\omega_{2}\right)} \\
E_{x\left(\omega_{1}\right)} E_{y\left(\omega_{2}\right)}+E_{y\left(\omega_{1}\right)} E_{x\left(\omega_{2}\right)}
\end{array}\right]
\end{aligned}
$$

Therefore the nonlinear polarization of SHG where $\omega_{3}=2 \omega$ is described as 


$$
\left[\begin{array}{l}
P_{x(2 \omega)} \\
P_{y(2 \omega)} \\
P_{z(2 \omega)}
\end{array}\right]=2 \varepsilon_{0}\left[\begin{array}{llllll}
d_{11} & d_{12} & d_{13} & d_{14} & d_{15} & d_{16} \\
d_{21} & d_{22} & d_{23} & d_{24} & d_{25} & d_{26} \\
d_{31} & d_{32} & d_{33} & d_{34} & d_{35} & d_{36}
\end{array}\right]\left[\begin{array}{c}
E_{x(\omega)}^{2} \\
E_{y(\omega)}^{2} \\
E_{z(\omega)}^{2} \\
2 E_{y(\omega)} E_{z(\omega)} \\
2 E_{x(\omega)} E_{z(\omega)} \\
2 E_{x(\omega)} E_{y(\omega)}
\end{array}\right]
$$

It is common to simplify this further by expressing this in terms of a $d_{\text {eff. }}$ The sumfrequency generation nonlinear polarization can be expressed as follows,

$$
P_{\left(\omega_{3}\right)}=4 \varepsilon_{0} d_{e f f} E_{\left(\omega_{1}\right)} E_{\left(\omega_{2}\right)}
$$

and for the case of SHG it is written as

$$
P_{(2 \omega)}=2 \varepsilon_{0} d_{e f f} E_{(\omega)}^{2}
$$

For example, crystalline quartz which is a crystalline form of silicon dioxide and is the second main constituent mineral compound of earth's crust belongs to the trigonal crystal system. It is a positive uniaxial crystal and is usually used as a standard in nonlinear optics experiments. Quartz In terms of its susceptibility tensor and crystal symmetry belongs to the crystal group of 32 . The tensor of this group has the following form

$$
\left[\begin{array}{cccccc}
d_{11} & d_{12} & 0 & d_{14} & 0 & 0 \\
0 & 0 & 0 & 0 & d_{25} & d_{26} \\
0 & 0 & 0 & 0 & 0 & 0
\end{array}\right]
$$


If we suppose there are two linearly polarized plane waves with amplitudes $E_{1}$ and $E_{2}$ propagating in a quartz crystal at an angle $\theta$, as shown in Fig. 3.1, to the optic axis (we can assume the optic axis is along the $\mathrm{Z}$ axis). In what follows we will consider the case where the sample is rotated around the Y-axis. In the cases we are concerned with the electric fields are generally oriented either in the k-Z plane (e-ray) or in the Y axis direction (oray). Therefore by using the above susceptibility tensor, the non-linear polarization at the sum frequency due to the interaction of these two fields is expressed as

$$
\begin{gathered}
P_{x\left(\omega_{3}\right)}=d_{11} E_{x\left(\omega_{1}\right)} E_{x\left(\omega_{2}\right)}+d_{12} E_{y\left(\omega_{1}\right)} E_{y\left(\omega_{2}\right)} \\
+d_{14} E_{y\left(\omega_{1}\right)} E_{z\left(\omega_{2}\right)}+d_{11} E_{z\left(\omega_{1}\right)} E_{y\left(\omega_{2}\right)} \\
P_{y\left(\omega_{3}\right)}=d_{25} E_{x\left(\omega_{1}\right)} E_{z\left(\omega_{2}\right)}+d_{25} E_{z\left(\omega_{1}\right)} E_{x\left(\omega_{2}\right)} \\
+d_{26} E_{x\left(\omega_{1}\right)} E_{y\left(\omega_{2}\right)}+d_{26} E_{y\left(\omega_{1}\right)} E_{x\left(\omega_{2}\right)} \\
P_{z\left(\omega_{3}\right)}=0
\end{gathered}
$$

Where according to the molecular structure of quartz and the crystal glass it belongs to (class 32 ), the nonlinear susceptibility matrix has the following symmetries

$$
\begin{aligned}
& d_{11}=-d_{12} \\
& d_{14}=-d_{25} \\
& d_{26}=-d_{11}
\end{aligned}
$$

Considering these symmetries we end up with: 


$$
\left[\begin{array}{cccccc}
d_{11} & -d_{11} & 0 & d_{14} & 0 & 0 \\
0 & 0 & 0 & 0 & -d_{14} & -d_{11} \\
0 & 0 & 0 & 0 & 0 & 0
\end{array}\right]
$$

For example if $E_{\left(\omega_{1}\right)}$ is an e-ray and $E_{\left(\omega_{2}\right)}$ is an o-ray then:

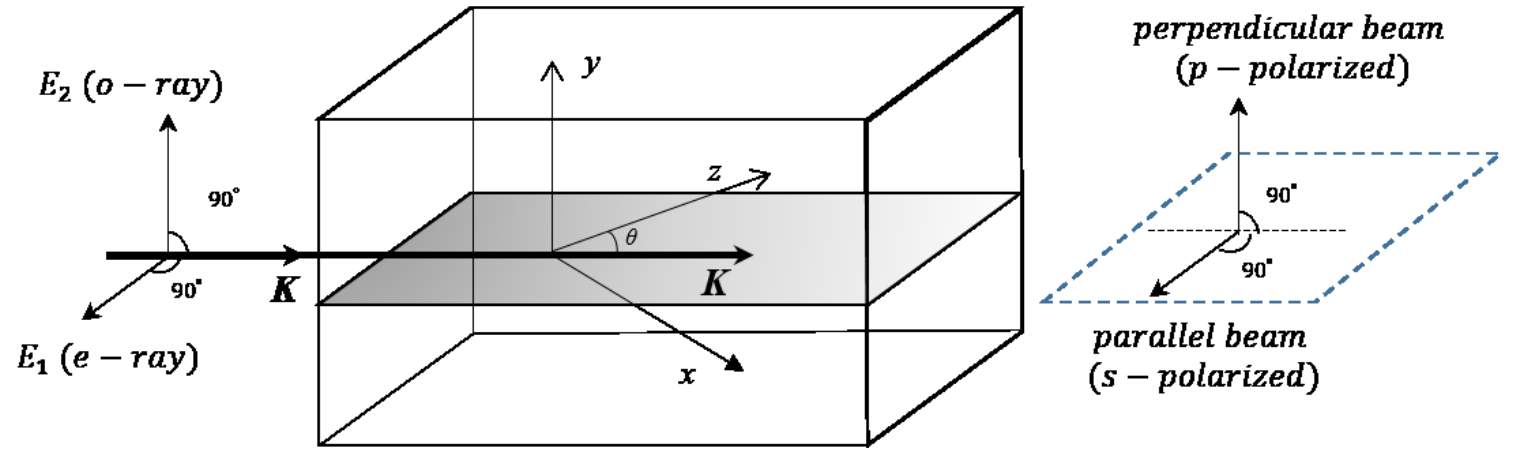

Figure 3.1. Principal plane of the crystal ( $k Z$ ) and: (a) ordinary beam. (b) extraordinary beam

$$
\begin{gathered}
E_{x\left(\omega_{1}\right)}=E_{\left(\omega_{1}\right)} \cos \theta \\
E_{y\left(\omega_{1}\right)}=0 \\
E_{z\left(\omega_{1}\right)}=E_{\left(\omega_{1}\right)} \sin \theta \\
E_{x\left(\omega_{2}\right)}=0 \\
E_{y\left(\omega_{2}\right)}=E_{\left(\omega_{2}\right)} \\
E_{z\left(\omega_{2}\right)}=0
\end{gathered}
$$

By substituting the equations we get 


$$
\begin{gathered}
P_{x\left(\omega_{3}\right)}=-E_{\left(\omega_{1}\right)} E_{\left(\omega_{2}\right)} d_{14} \sin \theta \\
P_{y\left(\omega_{3}\right)}=-E_{\left(\omega_{1}\right)} E_{\left(\omega_{2}\right)} d_{11} \cos \theta \\
P_{z\left(\omega_{3}\right)}=0
\end{gathered}
$$

If one was to measure the sum frequency light polarized perpendicular to the incident plane along the beam direction $\mathbf{K}$ (by placing a polarizer between the sample and the detector) then the measured field would be,

$$
P_{\perp}=P_{y}
$$

If one then measured the generated radiation in the plane of incidence (by inserting a polarizer between the sample and the detector) along the direction of the beam $\mathbf{K}$ then the measured light would have the polarization,

$$
\begin{gathered}
P_{\|}=P_{x} \cos \theta+P_{z} \sin \theta \\
P_{\|}=P_{x} \cos \theta
\end{gathered}
$$

Since $P_{z\left(\omega_{3}\right)}=0$.

Substituting in (3.51),

$$
\begin{gathered}
P_{\|}=-E_{\left(\omega_{1}\right)} E_{\left(\omega_{2}\right)} d_{14} \cos \theta \sin \theta \\
P_{\perp}=-E_{\left(\omega_{1}\right)} E_{\left(\omega_{2}\right)} d_{11} \cos \theta
\end{gathered}
$$


Since $=d_{e f f} E_{\left(\omega_{1}\right)} E_{\left(\omega_{2}\right)}$, therefore the resulting $d_{e f f}$ would be,

\section{Parallel case :}

$$
d_{e f f}=-d_{14} \cos \theta \sin \theta
$$

Perpendicular case :

$$
d_{\text {eff }}=-d_{11} \cos \theta
$$

For the case of our experiment both incident fields are in the $\mathrm{k}-\mathrm{Z}$ plane and the sample is being rotated only about the $\mathrm{Y}$ axis.

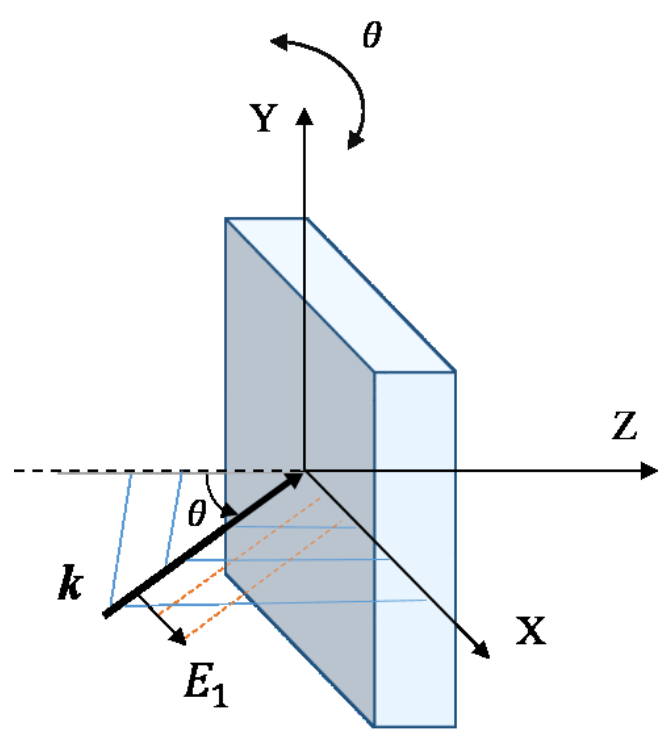

Figure 3.2. Sample being rotated

Similarly, the same procedure is performed to find the $d_{e f f}$ of poled silica which has the susceptibility tensor given by

$$
\left[\begin{array}{cccccc}
0 & 0 & 0 & 0 & d_{31} & 0 \\
0 & 0 & 0 & d_{31} & 0 & 0 \\
d_{31} & d_{31} & d_{33} & 0 & 0 & 0
\end{array}\right]
$$

For p-polarized case, the effective nonlinearity of poled silica is obtained as 


$$
d_{e f f}=3 d_{31} \sin \theta \cos ^{2} \theta+d_{33} \sin ^{3} \theta
$$

There is no s-polarized component to the field. If charge separation is principally responsible for the induced non-linearity (not the dipole orientation) then $d_{33}=3 d_{31}$. [27]

We have,

$$
\begin{gathered}
d_{e f f}=d_{33} \sin \theta \cos ^{2} \theta+d_{33} \sin ^{3} \theta \\
d_{e f f}=d_{33} \sin \theta\left(\cos ^{2} \theta+\sin ^{2} \theta\right)
\end{gathered}
$$

Thus

$$
d_{e f f}=d_{33} \sin \theta
$$

\subsection{Coupled-wave equations for sum-frequency and SHG}

\subsubsection{Sum-frequency}

This section shows how the amplitude of the generated sum frequency, $\omega_{3}$, wave varies as a result of its coupling to the input (fundamental) waves, $\omega_{1}$ and $\omega_{2}$. 


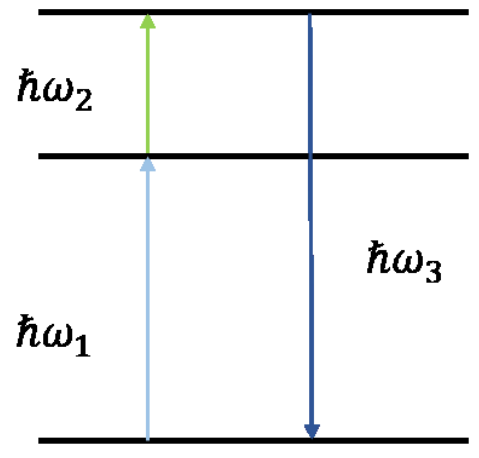

$\omega_{1}+\omega_{2}=\omega_{3}$

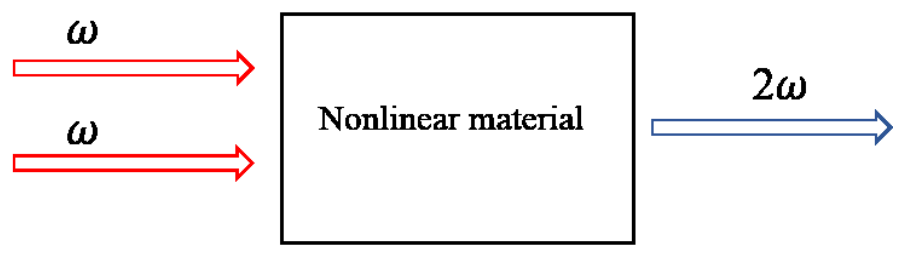

Figure 3.3. Sum frequency generation. Right diagram is for the case of second harmonic generation.

The nonlinear source term at the generated sum frequency, $\omega_{3}$, is represented as follows

$$
P_{3(z, t)}=P_{3} e^{-i \omega_{3} t}+c . c .
$$

We previously found for polarization in Eq. 3.42

$$
P_{\left(\omega_{3}\right)}=4 \varepsilon_{0} d_{e f f} E_{\left(\omega_{1}\right)} E_{\left(\omega_{2}\right)} \text { or more simply } P_{3}=4 \varepsilon_{0} d_{e f f} E_{1} E_{2}
$$

And for each input incident electric field

$$
E_{(z, t)}=E e^{-i \omega t}+c . c .
$$

Amplitude $E$ can be defined as an amplitude varying in space

$$
E=A e^{i k z} \quad \text { so } \quad E_{(z, t)}=A e^{i(k z-\omega t)}+c . c .
$$

By substituting the nonlinear polarization is found as 


$$
P_{3}=4 \varepsilon_{0} d_{e f f} E_{1} E_{2}=4 \varepsilon_{0} d_{e f f} A_{1} A_{2} e^{i\left(k_{1}+k_{2}\right) z}
$$

Substituting into wave equation (and assuming a plane wave)

$$
\frac{\partial^{2}}{\partial z^{2}} \mathbf{E}-\frac{\varepsilon_{(\omega)}^{(1)}}{c^{2}} \frac{\partial^{2}}{\partial t^{2}} \mathbf{E}=\frac{1}{\varepsilon_{0} c^{2}} \frac{\partial^{2}}{\partial t^{2}} \mathbf{P}
$$

We then can write

$$
\begin{gathered}
{\left[\frac{\partial^{2}}{\partial z^{2}} A_{3}+2 i k_{3} \frac{\partial}{\partial z} A_{3}-k_{3}^{2} A_{3}+\frac{\varepsilon_{\left(\omega_{3}\right)}^{(1)} \omega_{3}^{2} A_{3}}{c^{2}}\right] e^{i\left(k_{3} z-\omega_{3} t\right)}+c . c .} \\
=-4 \frac{d_{e f f} \omega_{3}^{2}}{c^{2}} A_{1} A_{2} e^{i\left[\left(k_{1}+k_{2}\right) z-\omega_{3} t\right)}+c . c .
\end{gathered}
$$

Knowing that $k_{3}^{2}=\frac{\varepsilon_{\left(\omega_{3}\right)}^{(1)} \omega_{3}^{2}}{c^{2}}$ and cancelling the frequency factor, the equation is then reduced to

$$
\frac{\partial^{2}}{\partial z^{2}} A_{3}+2 i k_{3} \frac{\partial}{\partial z} A_{3}=-4 \frac{d_{e f f} \omega_{3}^{2}}{c^{2}} A_{1} A_{2} e^{i\left(k_{1}+k_{2}-k_{3}\right) z}
$$

The summation and negation of all the input and output wave's propagation constants is called the wave-vector (momentum) mismatch and is commonly represented as,

$$
\Delta k=k_{1}+k_{2}-k_{3}
$$

Since the second derivative of the amplitude $A_{3}$ with respect to space, z, is much smaller than its first derivative therefore this term can be dropped and the equation is, 


$$
\frac{\partial}{\partial z} A_{3}=2 i \frac{d_{e f f} \omega_{3}^{2}}{k_{3} c^{2}} A_{1} A_{2} e^{i \Delta k z}
$$

The same procedure can be done to write the equation as the spatial variation of the input waves.

$$
\begin{aligned}
\frac{\partial}{\partial z} A_{1} & =2 i \frac{d_{e f f} \omega_{1}^{2}}{k_{1} c^{2}} A_{3} A_{2}^{*} e^{-i \Delta k z} \\
\frac{\partial}{\partial z} A_{2} & =2 i \frac{d_{e f f} \omega_{2}^{2}}{k_{2} c^{2}} A_{3} A_{1}^{*} e^{-i \Delta k z}
\end{aligned}
$$

\subsubsection{Second Harmonic Generation}

As was stated before for the case of second harmonic generation we can write nonlinear polarization as combination of the fundamental- and doubled- frequency polarizations.

$$
\mathbf{P}_{(z, t)}^{N L}=\mathbf{P}_{1(z, t)}+\mathbf{P}_{2(z, t)}
$$

Where $\mathbf{P}_{1(z, t)}$ and $\mathbf{P}_{2(z, t)}$ represent polarization of fundamental and SHG, respectively.

In terms of amplitude of waves, both polarization can be described as

$$
\begin{gathered}
P_{1(z)}=4 \varepsilon_{0} d_{e f f} E_{2} E_{1}^{*}=4 \varepsilon_{0} d_{e f f} A_{2} A_{1}^{*} e^{i\left(k_{2}-k_{1}\right) z} \\
P_{2(z)}=2 \varepsilon_{0} d_{e f f} E_{1}^{2}=2 \varepsilon_{0} d_{e f f} A_{1}^{2} e^{2 i k_{1} z}
\end{gathered}
$$


So coupled-amplitude equations for these two waves are expressed such

$$
\begin{gathered}
\frac{\partial}{\partial z} A_{1}=2 i \frac{d_{e f f} \omega_{1}^{2}}{k_{1} c^{2}} A_{2} A_{1}^{*} e^{-i \Delta k z} \\
\frac{\partial}{\partial z} A_{2}=i \frac{d_{e f f} \omega_{2}^{2}}{k_{2} c^{2}} A_{1}^{2} e^{i \Delta k z}
\end{gathered}
$$

Where

$$
\Delta k=2 k_{1}-k_{2}
$$

Equation (3.76) describes the generation of the second harmonic light through a non-linear medium for a continuous beam of light. This equation would be valid for the majority of Maker Fringe experiments that have been performed with pulses longer than 1 picosecond. As we will show, for femtosecond pulse lengths it is important to include the pulsed nature

of the field in the equation. Next, we will include the pulsed behavior in equation (3.76) to accurately model SHG with femtosecond pulses.

\subsection{Pulse Propagation}

A pulse can be described by two elements (functions), the pulse envelope function and a function that shows oscillation of the wave in space and time.

$$
E_{(z, t)}=A_{(z, t)} e^{i\left(k_{0} z-\omega_{0} t\right)}+c . c .
$$

In this equation $A_{(z, t)}$ is the pulse envelope function that propagates through the medium.

Propagation constant $k_{0}$ is written as, $k_{0}=\frac{n_{\left(\omega_{0}\right)} \omega_{0}}{c}$ 
We can start by writing the wave equation, where propagation is only along the $\mathrm{z}$ direction therefore the derivation of electric field with respect to space axes is only nonzero with respect to $\mathrm{z}$ component.

$$
\frac{\partial^{2}}{\partial z^{2}} E-\frac{1}{\varepsilon_{0} c^{2}} \frac{\partial^{2}}{\partial t^{2}} D=0
$$

To find the simplified form and solution for this equation it is easier to convert it to frequency domain by taking the Fourier transform of electric $\mathrm{E}$ and displacement $\mathrm{D}$ fields.

$$
\begin{aligned}
& E_{(z, t)}=\frac{1}{2 \pi} \int_{-\infty}^{\infty} E_{(z, \omega)} e^{-i \omega t} d \omega \\
& D_{(z, t)}=\frac{1}{2 \pi} \int_{-\infty}^{\infty} D_{(z, \omega)} e^{-i \omega t} d \omega
\end{aligned}
$$

Where $D_{(z, \omega)}$ is related to $E_{(z, \omega)}$ by

$$
D_{(z, \omega)}=\varepsilon_{0} \varepsilon_{(\omega)} E_{(z, \omega)}
$$

Now the Fourier transformed equation would be represented in frequency domain as

$$
\frac{\partial^{2}}{\partial z^{2}} E_{(z, \omega)}+\varepsilon_{(\omega)} \frac{\omega^{2}}{c^{2}} E_{(z, \omega)}=0
$$

Fourier transformed of $A_{(z, t)}$ is written as 


$$
A_{\left(z, \omega^{\prime}\right)}=\int_{-\infty}^{\infty} A_{(z, t)} e^{i \omega^{\prime} t} d t
$$

Electric field $E_{(z, \omega)}$ is related to $A_{(z, \omega)}$ as

$$
E_{(z, \omega)}=A_{\left(z, \omega-\omega_{0}\right)} e^{i k_{0} z}+A_{\left(z, \omega+\omega_{0}\right)}^{*} e^{-i k_{0} z}
$$

The second term on right hand side can be dropped as it rapidly varies with time, reducing the equation to

$$
E_{(z, \omega)} \simeq A_{\left(z, \omega-\omega_{0}\right)} e^{i k_{0} z}
$$

By replacing the electric field stated in terms of $A_{(z, \omega)}$ into

$$
\begin{aligned}
& \frac{\partial^{2}}{\partial z^{2}} E_{(z, \omega)}+\varepsilon_{(\omega)} \frac{\omega^{2}}{c^{2}} E_{(z, \omega)}=0 \\
& \frac{\partial^{2}}{\partial z^{2}} E_{(z, \omega)}=\left[\frac{\partial^{2}}{\partial z^{2}} A_{\left(z, \omega-\omega_{0}\right)}+i k \frac{\partial}{\partial z} A_{\left(z, \omega-\omega_{0}\right)}\right.+ \\
&\left.i k \frac{\partial}{\partial z} A_{\left(z, \omega-\omega_{0}\right)}-k^{2} A_{\left(z, \omega-\omega_{0}\right)}\right] e^{i k_{0} z}
\end{aligned}
$$

Since $A_{(z, \omega)}$ is a slowly varying function in space therefore its second derivative with respect to $\mathrm{z}$ is so small that can be ignored, therefore by knowing $k_{(\omega)}^{2}=\varepsilon_{(\omega)} \frac{\omega^{2}}{c^{2}}$ the final equation will be

$$
2 i k_{0} \frac{\partial}{\partial z} A+\left(k^{2}-k_{0}^{2}\right) A=0
$$


$k^{2}-k_{0}{ }^{2}=\left(k-k_{0}\right)\left(k+k_{0}\right), k$ is very close to $k_{0}$ therefore $\left(k+k_{0}\right)=2 k_{0}$ and approximately $k^{2}-k_{0}{ }^{2}=2 k_{0}\left(k-k_{0}\right)$, making the equation to be

$$
\frac{\partial}{\partial z} A_{\left(z, \omega-\omega_{0}\right)}-i\left(k-k_{0}\right) A_{\left(z, \omega-\omega_{0}\right)}=0
$$

Propagation constant $k$ can be described in the form of Taylor series expansion, because it depends on frequency.

Taylor Series:

$$
f_{(x)}=f_{(a)}+\frac{f_{(a)}^{\prime}}{1 !}(x-a)+\frac{f_{(a)}^{\prime \prime}}{2 !}(x-a)^{2}+\frac{f_{(a)}^{\prime \prime \prime}}{3 !}(x-a)^{3}
$$

\section{Propagation constant:}

$$
k=k_{0}+k_{1}\left(\omega-\omega_{0}\right)+\frac{1}{2} k_{2}\left(\omega-\omega_{0}\right)^{2}
$$

From the Taylor series we can find $k_{1}$ (the inverse of group velocity $v_{g}$ ) and $k_{2}$ (the dispersion of group velocity) as follow when $n_{\operatorname{lin}(\omega)}=\sqrt{\varepsilon_{(\omega)}}$

$$
k_{1}=\left(\frac{\partial k}{\partial \omega}\right)_{\omega=\omega_{0}}=\frac{1}{c}\left[n_{\operatorname{lin}(\omega)}+\omega \frac{\partial n_{\operatorname{lin}(\omega)}}{\partial \omega}\right]_{\omega=\omega_{0}}=\frac{1}{v_{g\left(\omega_{0}\right)}}
$$

In above the changes of refractive index with respect to frequency is minimal and can be omitted

$$
k_{2}=\left(\frac{\partial^{2} k}{\partial \omega^{2}}\right)_{\omega=\omega_{0}}=\frac{\partial}{\partial \omega}\left[\frac{1}{v_{g(\omega)}}\right]_{\omega=\omega_{0}}=\left(-\frac{1}{v_{g}^{2}} \frac{\partial v_{g}}{\partial \omega}\right)_{\omega=\omega_{0}}
$$


For expansion of the propagation constant equation the group velocity dispersion term can be ignored because of being very small in the experiments that we perform. Therefore $k$ can be expressed as

$$
k=k_{0}+k_{1}\left(\omega-\omega_{0}\right)
$$

Now the expression for $k$ is inserted into the reduced wave equation.

$$
\frac{\partial}{\partial z} A-i k_{1}\left(\omega-\omega_{0}\right) A=0
$$

Then this equation is transformed back to time domain by applying inverse Fourier transform.

$$
A_{(z, t)}=\frac{1}{2 \pi} \int_{-\infty}^{\infty} A_{\left(z, \omega-\omega_{0}\right)} e^{-i\left(\omega-\omega_{0}\right) t} d\left(\omega-\omega_{0}\right)
$$

Taking derivative of $A$ with respect to time we obtain

$$
\begin{gathered}
\frac{\partial}{\partial t} A_{(z, t)}=\frac{1}{2 \pi} \int_{-\infty}^{\infty}\left(\omega-\omega_{0}\right) A_{\left(z, \omega-\omega_{0}\right)} e^{-i\left(\omega-\omega_{0}\right) t} d\left(\omega-\omega_{0}\right) \\
i \frac{\partial}{\partial t} A_{(z, t)}=\frac{1}{-i} \frac{1}{2 \pi} \frac{\partial}{\partial t} \int_{-\infty}^{\infty} A_{\left(z, \omega-\omega_{0}\right)} e^{-i\left(\omega-\omega_{0}\right) t} d\left(\omega-\omega_{0}\right)
\end{gathered}
$$

Hence, 


$$
\frac{\partial}{\partial z} A+k_{1} \frac{\partial}{\partial t} A=0
$$

\subsection{Time-dependent SHG coupled mode equations}

If we combine the results from 3.4.2 (Eq. (3.76)) and 3.5 (Eq. (3.99)) we end up with the time dependent equation for the SHG in a non-linear poled silica sample,

$$
\frac{\partial}{\partial z} A_{2}+k_{1,2 \omega} \frac{\partial A_{2}}{\partial t}=i \frac{d_{e f f} \omega_{2}^{2}}{k_{o, 2 \omega} c^{2}} A_{1}^{2} e^{i \Delta k z}
$$

A few more elements need to be factored in before we will arrive at a final equation that describes the multi-layer maker fringe experiments.

In order to avoid confusion, it must be mentioned that $\left(k_{2}=k_{1,2 \omega}\right)$ and $\left(k_{1}=k_{1, \omega}\right)$

\subsection{Moving frame of reference of time $\tau$}

We have our current time-dependent equation for the SHG in a poled structure:

$$
\frac{\partial}{\partial z} A_{2}+k_{1,2 \omega} \frac{\partial A_{2}}{\partial t}=i \frac{d_{e f f} \omega_{2}^{2}}{k_{o, 2 \omega} c^{2}} A_{1}^{2} e^{i \Delta k z}
$$


By applying coordinate transformation and time retardation, the equation can be simplified to travel in the frame of reference of the fundamental beam. Time shifting can be expressed as

$$
\tau=t-\frac{Z}{v_{g}}=t-k_{1} z
$$

The new time shifted optical pulse function is related to the original pulse as follow

$$
A_{s(z, \tau)}=A_{(z, t)}
$$

The new pulse is then substituted in the above stated equation. To do the derivation it is required to use the chain rule

$$
\begin{gathered}
\frac{\partial A}{\partial z}=\frac{\partial A_{s}}{\partial z}+\frac{\partial A_{s}}{\partial \tau} \frac{\partial \tau}{\partial z}=\frac{\partial A_{s}}{\partial z}-k_{1, \omega} \frac{\partial A_{s}}{\partial \tau} \\
\frac{\partial A}{\partial t}=\frac{\partial A_{s}}{\partial z} \frac{\partial z}{\partial t}+\frac{\partial A_{s}}{\partial \tau} \frac{\partial \tau}{\partial t}=\frac{\partial A_{s}}{\partial \tau} \\
\frac{\partial^{2} A}{\partial t^{2}}=\frac{\partial^{2} A_{s}}{\partial \tau^{2}}
\end{gathered}
$$

Substituting into the equation we get

$$
\frac{\partial}{\partial z} A_{2}+k_{1,2 \omega} \frac{\partial A_{2}}{\partial t}=i \frac{d_{e f f} \omega_{2}^{2}}{k_{o, 2 \omega} c^{2}} A_{1}^{2} e^{i \Delta k z}
$$




$$
\begin{gathered}
\left(\frac{\partial A_{s}}{\partial z}-k_{1, \omega} \frac{\partial A_{s}}{\partial \tau}\right)+k_{1,2 \omega}\left(\frac{\partial A_{s}}{\partial \tau}\right)=i \frac{d_{e f f} \omega_{2}^{2}}{k_{o, 2 \omega} c^{2}} A_{1}^{2} e^{i \Delta k z} \\
\frac{\partial A_{s}}{\partial z}+\left(k_{1,2 \omega}-k_{1, \omega}\right) \frac{\partial A_{s}}{\partial \tau}=i \frac{d_{e f f} \omega_{2}^{2}}{k_{o, 2 \omega} c^{2}} A_{1}^{2} e^{i \Delta k z}
\end{gathered}
$$

Since,

$$
i \frac{d_{e f f} \omega_{2}^{2}}{k_{1,2 \omega} c^{2}} A_{1}^{2} e^{i \Delta k z}=i \frac{d_{e f f} \omega_{2}}{n_{2 \omega} c} A_{1}^{2} e^{i \Delta k z}
$$

Then,

$$
\frac{\partial A_{2}}{\partial z}+\left(k_{1,2 \omega}-k_{1, \omega}\right) \frac{\partial A_{2}}{\partial \tau}=i \frac{d_{e f f} \omega_{2}}{n_{2 \omega} c} A_{1}^{2} e^{i \Delta k z}
$$

This equation describes the generation of second harmonic light in our sample where $A_{2}$ is the electric field amplitude of the second harmonic light and $A_{1}$ is the amplitude of the fundamental radiation. The frame of reference for the model moves with the fundamental pulse. 


\section{Chapter 4: Maker Fringes}

\subsection{History and Method}

At an early stage in the development of non-linear optical components it was known from theory that ideal material for SHG would be a non-centrosymmetric crystal. Moreover, the crystal had to be transparent for all frequencies in the interaction. A material that is very abundant and transparent in the visible and near-infrared is quartz. Franken and his colleagues were the first to observe a small SHG signal using a Q-switched ruby laser, filters and a spectrograph [29].

To measure the nonlinearity of a material, a good comparative measurement technique was introduced by Maker et al in 1962 [4], and was named after him as the Maker fringe method. In this method, described in section 2.4 , the SHG generation produced by a sample is compared to a known reference, such as quartz.

In SHG the fundamental and the $\mathrm{SH}$ waves do not travel at the same velocities (as a result of material dispersion), the SH waves generated at different locations within the nonlinear material are not always in phase - at some point, destructive interference of the SH waves causes a decrease in the generated $\mathrm{SH}$ and the power flows back into the fundamental wave. The periodicity of this effect is quantified by a parameter known as the coherence length, which is essentially a measure of the maximum "useful" length of the nonlinear material. In the Maker fringe experiment the sample is rotated around the Y-axis. This essentially increases the path length that the light must travel through to exit the sample. 


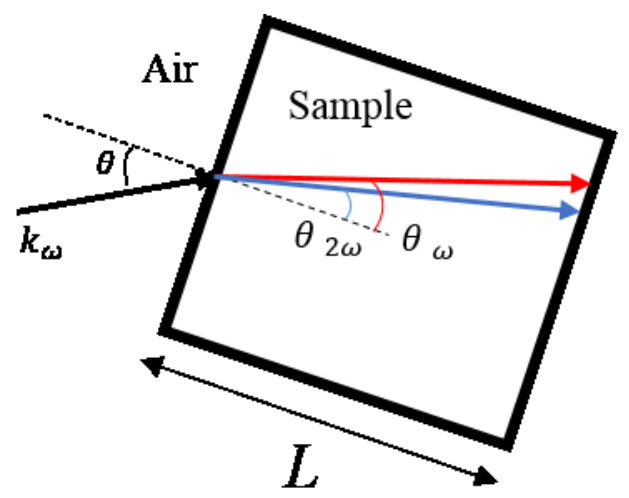

Figure 4.1. Propagation of the fundamental and the second harmonic waves during a Maker fringe measurement.

There is a small discrepancy in angle between the fundamental and second harmonic, however, this is typically quite small and has not been considered in our experiments. The phase matching term for describing the overlap between fundamental and SHG in the sample is

$$
\Delta k L=\left(n_{(2 \omega)}-n_{(\omega)}\right) \frac{2 \omega}{c} L
$$

The coherence length can be defined as the length over which the above equation is equivalent to $\pi$ ( $2 \pi$ is sometimes considered the coherence length as well). In a Maker fringe study $L$ is increased as the sample rotates by a factor of $L / \cos \theta$.

As the sample is rotated the path length increases. For every multiple of the coherence length the SHG will go from a maximum to a minimum. Several of these peaks appear as 'fringes', hence the name of the measurement technique. To account for the increasing path length this $1 / \cos \theta$ dependence has been incorporated into our model. 
Furthermore in order to account for the beam reflection and refraction we need to consider the Fresnel transmission equations. The first reflection occurs for the fundamental beam at the entrance interface (air-sample interface) and the second reflection occurs for the second harmonic wave at exit interface (sample -air interface). General Fresnel equation of transmittance is expressed as,

$$
T=\frac{n_{2} \cos \theta_{t}}{n_{1} \cos \theta_{i}}|t|^{2}
$$

Where $\theta_{t}$ is the angle the transmitted beam (in second medium) makes with the normal of interface, and $\theta_{i}$ is the angle of incident beam (in first medium). Hence for fundamental beam at the front interface the transmittance $T$ is expressed as

$$
T_{\omega}=\frac{n_{\omega} \cos \theta_{\omega}}{\cos \theta}\left|t_{a \rightarrow s}\right|^{2}
$$

Where standard Fresnel transmission term for the air-sample interface, $t_{a \rightarrow s}$, for the two different polarizations of the signal are given as,

$$
\begin{aligned}
& t_{s}=\frac{2 n_{1} \cos \theta_{i}}{n_{1} \cos \theta_{i}+n_{2} \cos \theta_{t}} \\
& t_{p}=\frac{2 n_{1} \cos \theta_{i}}{n_{1} \cos \theta_{t}+n_{2} \cos \theta_{i}}
\end{aligned}
$$

Which for our case would be written as

$$
t_{a \rightarrow s}^{s-p o l}=\frac{2 \cos \theta}{\cos \theta+n_{\omega} \cos \theta_{\omega}}
$$




$$
t_{a \rightarrow s}^{p-p o l}=\frac{2 \cos \theta}{\cos \theta_{\omega}+n_{\omega} \cos \theta}
$$

And at back of the sample (sample-air interface) the transmittance for the second harmonic beam is given as

$$
T_{2 \omega}=\frac{\cos \theta}{n_{2 \omega} \cos \theta_{2 \omega}}\left|t_{s \rightarrow a}\right|^{2}
$$

And Fresnel transmission term at sample-air interface for two polarizations for SHG is

$$
\begin{aligned}
& t_{s \rightarrow a}^{s-p o l}=\frac{2 n_{2 \omega} \cos \theta_{2 \omega}}{n_{2 \omega} \cos \theta_{2 \omega}+\cos \theta} \\
& t_{s \rightarrow a}^{p-p o l}=\frac{2 n_{2 \omega} \cos \theta_{2 \omega}}{n_{2 \omega} \cos \theta+\cos \theta_{2 \omega}}
\end{aligned}
$$

Since in our experiment we are only dealing with p-polarized beam so we only consider the p-polarized transmission term.

For a uniform medium we know that second harmonic filed in terms of the fundamental is expressed as follow passing through a sample of thickness $L$ with uniform medium,

$$
E_{2 \omega}(z)=\epsilon_{0} \frac{-j \omega}{2} \sqrt{\frac{\mu_{0}}{\epsilon}} E_{\omega}^{2} \chi_{e f f} \frac{e^{j \Delta k L}-1}{j \Delta k}
$$

And intensity is related to square of the field by 


$$
I=\frac{|E|^{2}}{2 \sqrt{\frac{\mu_{0}}{\epsilon}}}
$$

So the second harmonic intensity can be written as

$$
I_{2 \omega}=\epsilon_{0}^{2} \frac{\omega^{2} L^{2}}{2 n_{\omega}^{2} n_{2 \omega}}\left(\frac{\mu_{0}}{\epsilon_{0}}\right)^{\frac{3}{2}} \chi_{e f f}^{2} I_{\omega}^{2}\left[\frac{\sin \left(\frac{\Delta k L}{2}\right)}{\frac{\Delta k L}{2}}\right]^{2}
$$

Which becomes

$$
I_{2 \omega}=\frac{\omega^{2} L^{2}}{2 \epsilon_{0} c^{3} n_{\omega}^{2} n_{2 \omega}} \chi_{e f f}^{2} I_{\omega}^{2}\left[\operatorname{sinc}\left(\frac{\Delta k L}{2}\right)\right]^{2}
$$

Considering the effect of Fresnel transmission on two interfaces of sample for fundamental and second harmonic beam and also the substituting for effective second order nonlinearity (Eq. 3.55), we get

$I_{2 \omega}=\frac{2 \omega^{2} L^{2}}{\epsilon_{0} c^{3} n_{\omega}^{2} n_{2 \omega}}\left(d_{33} \sin \theta\right)^{2} I_{\omega}^{2}\left[\operatorname{sinc}\left(\frac{\Delta k L}{2}\right)\right]^{2}\left[T_{\omega(a \rightarrow s)}^{2}(\theta)\right]\left[T_{2 \omega(s \rightarrow a)}\right]$

The detector measures the power of the output (SHG) beam (which is linearly and directly proportional to intensity) therefore in the model code the output second harmonic beam was multiplied by the Fresnel transmission factors just as the above equation to exactly simulate the real experiment. 


\section{Chapter 5: Split-Step Fourier Method}

\subsection{Theory and equations}

In our research, to model the pulse propagation through the sample, we employed a Split-Step Fourier Method (SSFM) which is an efficient numerical technique that is commonly used for the problems involving optical wave propagation.

The equation that we have for our model can be separated into two distinct parts. There is a term that describes the propagation of the generated second harmonic through the medium and a term that describes the generation of the radiation. This is shown in the equation below,

$$
\begin{gathered}
\frac{\partial A_{2}}{\partial z}+\left(k_{1,2 \omega}-k_{1, \omega}\right) \frac{\partial A_{2}}{\partial \tau}=i \frac{d_{e f f} \omega_{2}}{n_{2 \omega} c} A_{1}^{2} e^{i \Delta k z} \\
\frac{\partial A_{2}}{\partial z}=-\left(k_{1,2 \omega}-k_{1, \omega}\right) \frac{\partial A_{2}}{\partial \tau}+i \frac{d_{e f f} \omega_{2}}{n_{2 \omega} c} A_{1}^{2} e^{i \Delta k z} \\
\frac{\partial A_{2}}{\partial z}=(\hat{P}+\widehat{N}) A_{2}
\end{gathered}
$$

Where $\hat{P}$ is a differential operator that accounts for the pulse propagation in the medium (in the frame of reference of the fundamental) and $\widehat{N}$ is a nonlinear operator that accounts for the generation of the second harmonic. Generally, the second harmonic radiation is generated and lags behind the fundamental radiation in a continuous fashion as its group 
velocity is less than that of the red light. The SSFM assumes that this can be discretized over small distances (h) so that the non-linear term generates the second harmonic and the generated pulse shifts in time in alternating steps. This means that in one step only the non-linear term acts followed by a step where only the propagation term acts. The nonlinear term is as follows:

Step 1

$$
\frac{\partial A_{2}}{\partial z}=i \frac{d_{e f f} \omega_{2}}{n_{2 \omega} c} A_{1}^{2} e^{i \Delta k z}
$$

This is converted into a difference equation, which can be solved easily for $A_{2}(z+h)$

$$
\frac{A_{2}(z+h)-A_{2}(z)}{\Delta z}=i \frac{d_{e f f} \omega_{2}}{n_{2 \omega} c} A_{1}^{2} e^{i \Delta k z}
$$

The propagation term is then determined by the equation:

Step 2

$$
\frac{\partial A_{2}}{\partial z}=-\left(k_{1,2 \omega}-k_{1, \omega}\right) \frac{\partial A_{2}}{\partial \tau}
$$

To solve this we apply a fourier transform to both sides of the equation and solve for $\mathrm{A}_{2}$ (assuming that the time derivative in the frequency domain means multiplications by -i $\omega$ ),

$$
\begin{gathered}
\frac{\partial A_{2}}{\partial z}=i \omega\left(k_{1,2 \omega}-k_{1, \omega}\right) A_{2} \\
A_{2(\omega)}(z+h)=e^{i \omega\left(k_{1,2 \omega}-k_{1, \omega}\right) h} A_{2(\omega)}(z)
\end{gathered}
$$


This equation represents a shift in the time domain. For the discrete case (since we employ a FFT) the frequency is written as $\omega=2 \pi n / N \Delta$. Here $n / N \Delta$ ranges from $-1 / 2$ to $1 / 2$. In the program $\mathrm{n}$ is equivalent to $\mathrm{t}$ and $\mathrm{N}$ is the total number of samples in $\mathrm{t}$. With these substitutions (5.7) becomes,

$$
A_{2(\omega)}(z+h)=e^{i 2 \pi\left(k_{1,2 \omega}-k_{1, \omega}\right) \frac{h t}{N \Delta}} A_{2(\omega)}(z)
$$

In the program t ranges from -10000 to 10000 with a delta of 0.1 and $\mathrm{N}$ is 20001 .

Step 1 and Step 2 alternate to numerically calculate the solution for Eq. (3.111). This method allows for the SHG from an arbitrary non-linearity to be modeled.

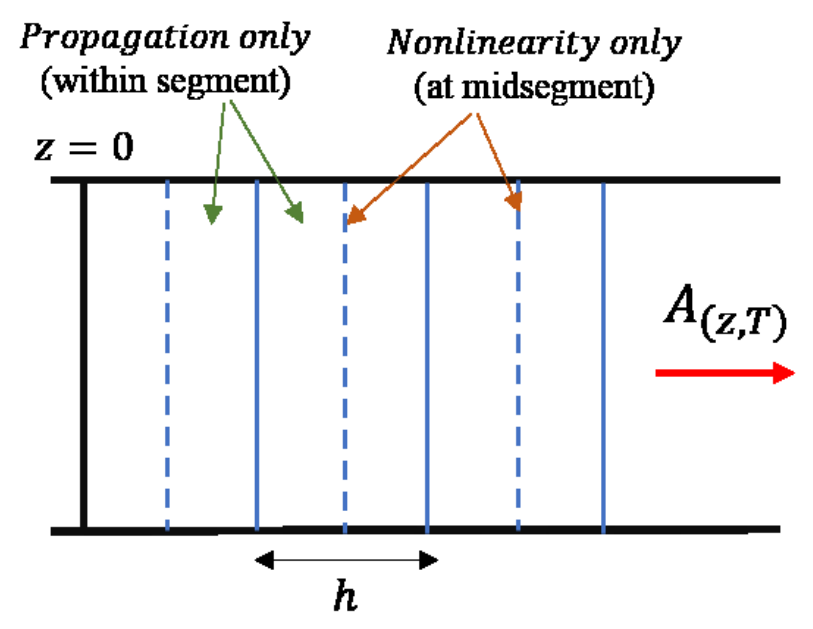

Figure 5.1. Split-Step Fourier Method. The propagation and non-linearity act in alternating steps. 


\section{Chapter 6: Experiment}

\subsection{Femtosecond Maker Fringes: Time Domain Study}

Maker fringe experiments are usually performed by using picosecond or nanosecond pulsed lasers which are longer than femtosecond laser pulses.

The maker fringe pattern for the following multilayer structure using the picosecond (in theory) and femtosecond pulsed lasers are shown below in figures 6.2 and 6.3 respectively [25]. The source of fundamental beam used to generate Fig. 6.3 was an ultrafast modelocked Ti:sapphire laser (operating wavelength $800 \mathrm{~nm}$, pulse width $<70 \mathrm{fs}$, repetition rate $80 \mathrm{MHz}$ ). Maker Fringes were generated by illuminating the sample with a p-polarized beam as it was rotated from $-80^{\circ}$ to $80^{\circ}$ in the plane of incidence (relative to the normal of the sample and incident laser beam). The generated second harmonic light was captured by a photomultiplier tube (PMT) after passing through a bandpass filter.

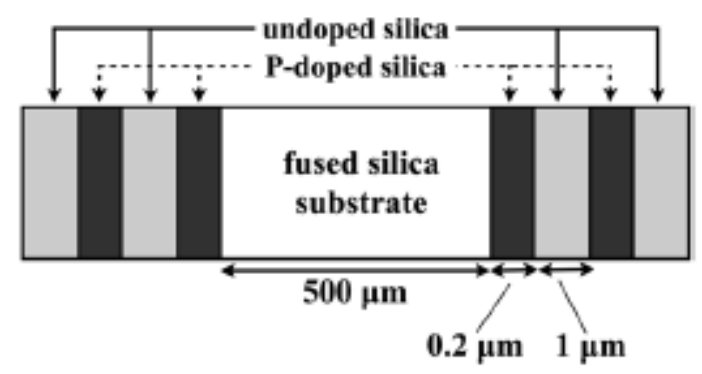

Figure 6.1. The multi-layer stacks were on either side of the substrate. 


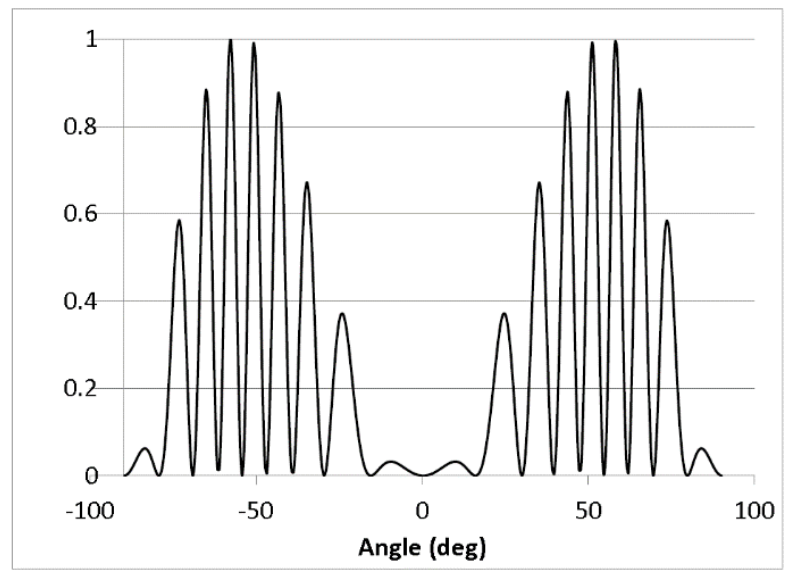

Figure 6.2. Theoretical Maker Fringe pattern for the sample in Fig. 6.1 considering a long picosecond length fundamental pulse.

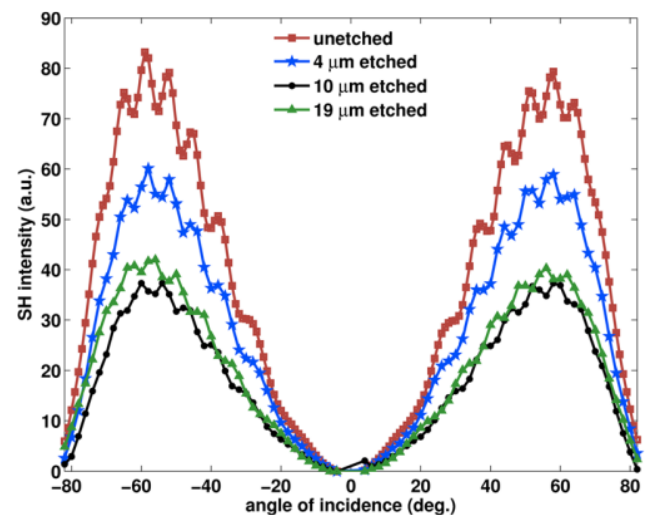

Figure 6.3. Maker Fringe patterns with femtosecond pulses. The impact of etching away the multi-layer stack is also shown.

It is seen that fringe visibility of the experiment is much lower than the theoretical pattern.

The reason for this could be that the nonlinearity is mostly accumulated on one side of the sample although, when etched, the non-linearity on either side of the sample was equivalent as can be seen in Fig. 6.3 (etching on one side reduces the SHG by a factor of 2).

A theoretical model of pulse propagation, using a split-step Fourier method, was developed to simulate short pulse SHG in an effort to determine the origin of the reduced visibility 
(smaller fringes) in the pattern. The equation that models the pulsed generation of SHG is Eq. 3.111, reproduced again here.

$$
\frac{\partial A_{2}}{\partial z}+\left(k_{1,2 \omega}-k_{1, \omega}\right) \frac{\partial A_{2}}{\partial \tau}=i \frac{d_{e f f} \omega_{2}}{n_{2 \omega} c} A_{1}^{2} e^{i \Delta k z}
$$

Where, again, $A_{2}$ is the amplitude of the second harmonic wave, $A_{1}$ is the amplitude of fundamental beam, $k_{1}$ is the inverse of the group velocity, $\omega$ is the angular frequency of the fundamental beam, $d_{e f f}$ is the effective nonlinearity and $\Delta k$ is the phase matching term $\Delta k=k_{2}-k_{1}$. Solving (6.1) with the SSFM, for the above-depicted sample (Fig. 6.1) and an input temporal pulse length of 80 femtoseconds, it was possible to simulate the reduced fringe visibility seen in Fig. 6.3.

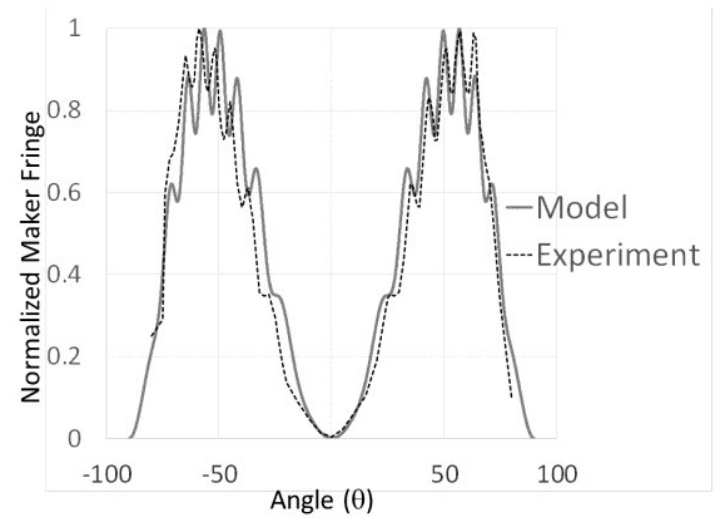

Figure 6.4. Modeled Maker Fringe pattern for the sample in Fig. (6.1) considering an 80 femtosecond fundamental pulse.

The reason for the reduced fringe visibility, at least for a continuous non-linearity in a sample, can be linked to the behavior of pulse propagation through the sample. As the pulse enters the sample, second harmonic light is generated by the fundamental pulse. Since the 
group velocity of the SH light is smaller than that of fundamental pulse, it lags behind. Consequently each SH pulse that is generated later in the sample will be leading the pulses which are generated earlier in the sample. Because only a portion of the pulses overlap, perfect destructive interference of the SHG cannot occur. This lack of destructive interference means that the visibility of the Maker fringe is compromised. This mechanism can be seen below.

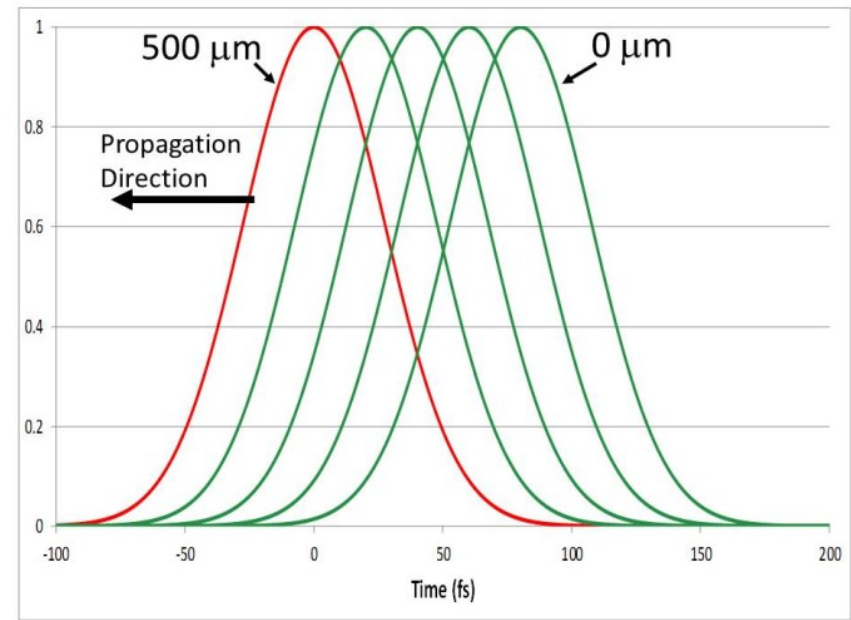

Figure 6.5. The mechanism for the reduction in fringe visibility with $f$ f fundamental pulse lengths. The green light (doubled frequency, SH) produced at different points in the material does not overlap well on account of the discrepancy in group velocity between the fundamental and second harmonic radiation.

In this figure the left most pulses represent the fundamental light which is leading the other created SH pulses. The right most pulse is the light generated at the entrance of the sample or in other words the very first SH created into the sample, the other green pulses on the right of this pulse which are leading were generated later in the sample. After the pulses exit the sample, they are added and time-averaged at the detector. For long fundamental pulses (nanosecond and picosecond) the created SH pulses at different points of the sample 
have nearly complete overlap with one another thus the discrepancy in group velocity does not affect the fringes. For shorter pulses adjacent pulses to cease to overlap.

In the case of the sample shown in Fig. 6.1, light is only generated at the beginning and end of the sample. The pulse overlap is shown in Fig. 6.6.

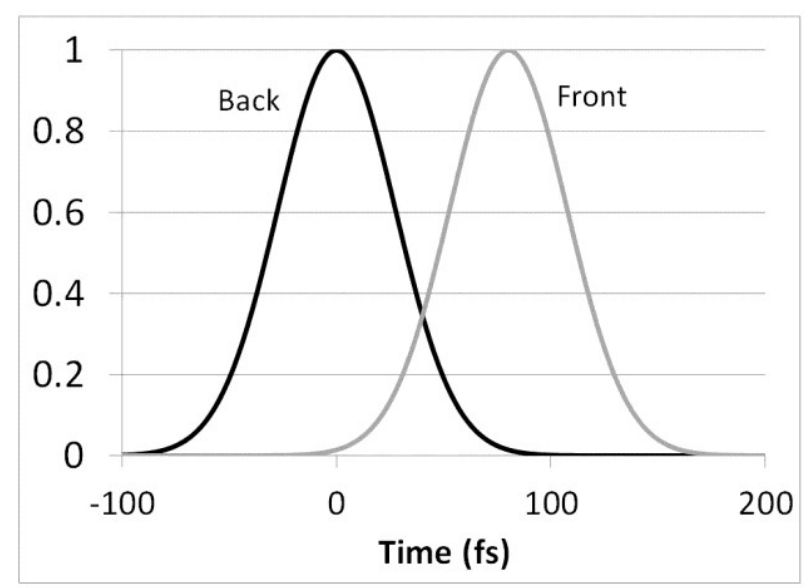

Figure 6.6. Second Harmonic light generated at either side of the sample. Clearly, there is very little overlap between the pulses.

From this figure it is clear that there is very little overlap between the light generated at the front and back of the sample. This would result in diminished fringe visibility. 


\subsection{Variation of spacing and number of layers}

To further study the multilayered structures in an effort to enhance the magnitude of generated SH beam, we focused on the effect of spacing and number of layers on generated light. This work relates to the second set of experiments performed by Ksenia Yadav [26].

The configuration for this experiment had layers only on one side. One was a structure with two Ge-doped layers separated by 1 micron and a total thickness of $3 \mu \mathrm{m}$. The other one was consisting of 40 layers separated by $75 \mathrm{~nm}$ for a total thickness the same as the previous, $3 \mu \mathrm{m}$. These two samples were compared to unlayered bulk silica sample.

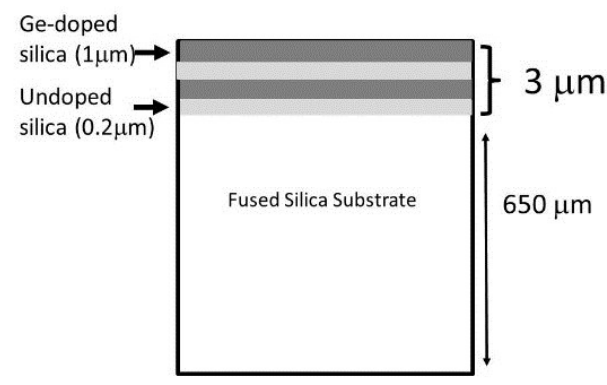

Ge content: 22 wt\%

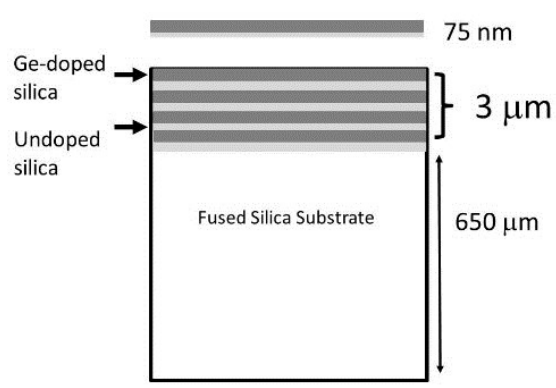

Ge content: 0.2 wt $\%$

Figure 6.7. The two samples with equal thickness but different separation.

These samples were poled using the contact poling technique in which the sample was placed between two n-type electrodes with voltage of $3000 \mathrm{~V}$ at $300{ }^{\circ} \mathrm{C}$ for duration of 8 minutes then the heat was turned off and the sample was allowed to cool down for 1.5 hours after which the voltage was switched off. Maker Fringe measurements showed that the generated second harmonic in the 40 layered sample was 204 times greater than that in a bulk silica sample, and approximately 80 times greater than SHG produced in the 2 layered structure. If we assume the increase in observed SHG was a consequence of an 
increase in induced nonlinearity, $\chi^{(2)}$, this means a 14 -fold $(\sqrt[2]{208} \approx 14)$ increase in the nonlinearity of the 40-layer structure relative to pure silica. This was not probable so the more reasonable explanation would be due to introducing a number of layers (interfaces) and nonlinear regions within the sample. The influence of spacing width on the SHG measurement was not considered in that study.

In order to model the generation of SHG in these types of structures, since the thickness of sample is only $3 \mu \mathrm{m}$, the discrepancy in group velocity is not considered in the model equation and the pulsed nature of the beam is ignored. Thus the reduced form of our main equation for SHG in this case would be:

$$
\frac{\partial A_{2}}{\partial z}=-\frac{i \omega}{n_{2 \omega} c} d_{e f f} A_{1}^{2} e^{i \Delta k z}
$$

To study the impact of separation and number of layers, two possible scenarios were investigated.

-What happens when the layer spacing is increased.

-What happens when both the spacing is increased and the number of layers is reduced. This second model could then be compared to the Ksenia's experiment.

These scenarios can be depicted as follows, 


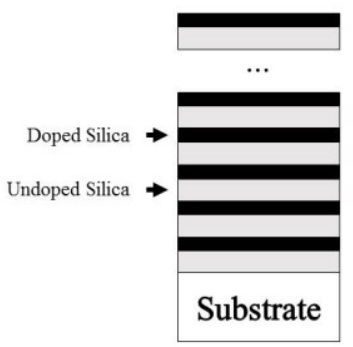

(a)

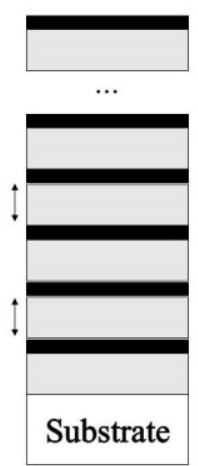

(b)

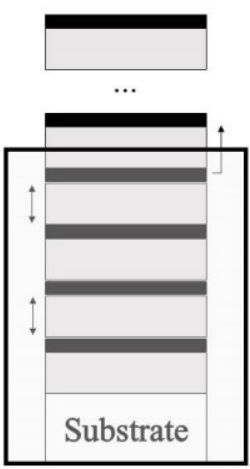

(c)

Figure 6.8. Three structures were compared in our model. (a) 40-layer structure. (b) Stretched 40 layer structure (Separation increasing). (c) Stretched structure with constant length (separation increasing, number of layers reducing consequently).

For these structures and model it was assumed that all layers possess equal nonlinearity and that the separation between non-linear regions could be as small as $75 \mathrm{~nm}$ (although in practice it is not possible to measure the nonlinearity width smaller than $500 \mathrm{~nm}$ using a Maker Fringe configuration [35]). These assumptions are not certain and it is very probable that one or both assumptions may be invalid in practice. The standard and reference structure in this experiment is the one shown on figure 6.9 (a), which is $3 \mu \mathrm{m}$ thick and is composed of 40 layers (of doped silica) separated by $75 \mathrm{~nm}$ (of un-doped silica).

Since the scale of these structures are very small, and it was found in previous experiments that nonlinearity occurs in a very thin region at the interface between layers, in our model we assumed each nonlinear region to be a point source.

In the first scenario we were interested to see the impact of increasing the spacing or interval between each of the 40 layers. To do so we set the initial separation to be $75 \mathrm{~nm}$ (as the reference structure) and increased it in increments to the maximum separation of 25 
$\mu \mathrm{m}$. The maximum peak of SHG produced from Maker fringe experiment at each increment was then calculated and normalized to the SHG of reference sample (a), this is shown in Fig. 6.10. As can be seen in the figure, the observed SHG drops very rapidly as the layer separation increases. This drop is due to destructive interference of the second harmonic lights generated at the layers. It is clear in the graph that as the spacing approaches the coherence length between the fundamental and second harmonic, the interference comes back into phase and the SHG recovers to the peak.

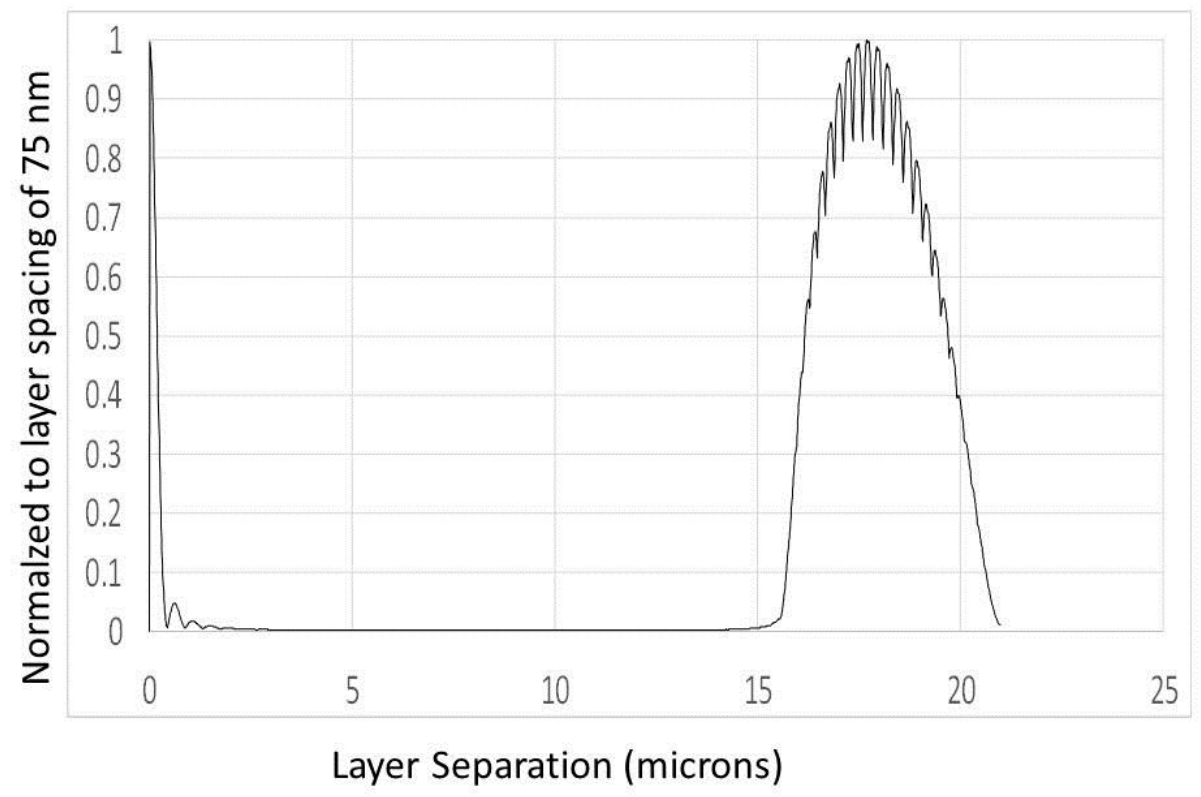

Figure 6.9. SHG of sample (b) normalized to SHG of (a). We see that the SHG dramatically decreases with layer spacing.

SHG is very sensitive to the variation of separation and significantly altered with increasing layer spacing as it is evident from the figure. 
The second scenario was similar to the first scenario with one difference in which a limit was set for the overall length of the varying-length structure. In this manner the length of the sample was fixed to a constant value so the variation in spacing would result in variation in number of layers such that as the separation increased the number of layers would reduce. This can be visualized as a box of fixed length that as each successive layer goes out of the box it would be removed from the calculation as shown in Fig. 6.9 (c). In Fig. 6.11 we see the ratio of the SHG light the model predicts for sample 6.9 (a) over that for 6.9 (c) as a function of layer spacing.

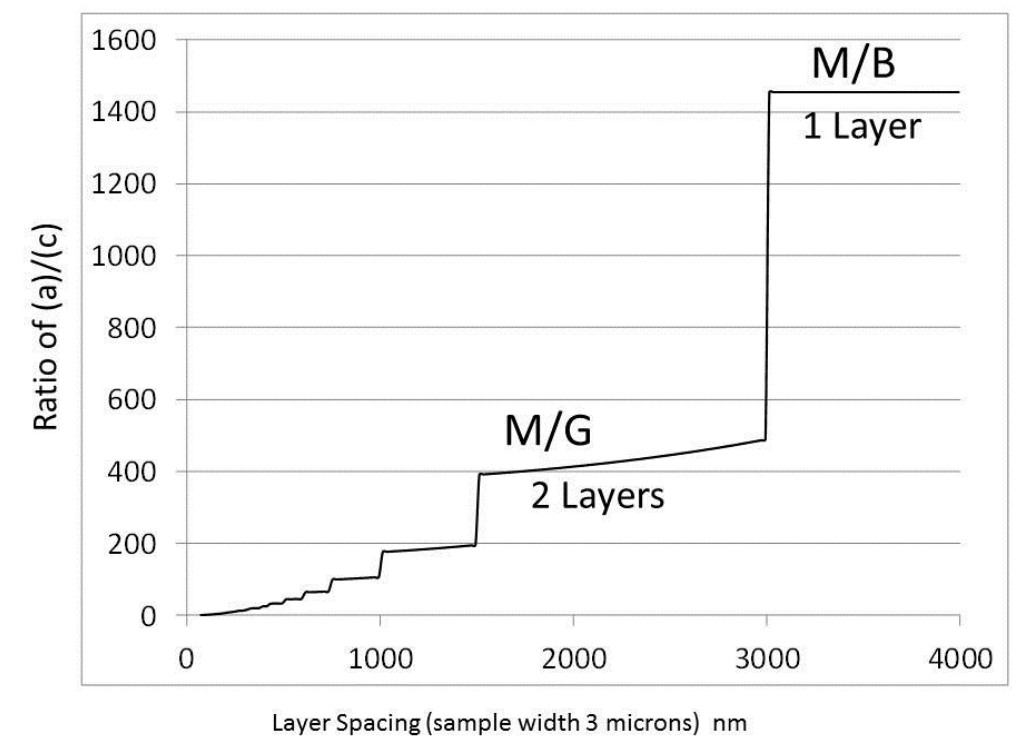

Figure 6.10. Ratio of SHG generated by the 40 layered structure (a) to that generated with (c) from Fig. 6.9.

. We see that the ratio evolves in a step-like fashion as the separation increases, on account of the elimination of one more layer. Finally, we are left with only 1 layer (labeled on the graph). We see that 1 and 2 layer structures generate $\sim 1450$ and $\sim 400$ less SHG than the 40 layer structure, respectively. This is very close to the expected value as 40 layers, 
exactly in phase, should produce $40^{2}=1600$ times the SHG of a single layer. The slight reduction is a result of the incomplete constructive interference in the 40-layer sample (it is not perfectly continuous as there are $75 \mathrm{~nm}$ between layers).

The ratios produced by the model are clearly not equivalent to the 204 and 80 ratio observed in the experiment. There are several possible explanations for this discrepancy. First, it was assumed that the non-linearity generated at each layer was equivalent in magnitude to that produced in a bulk sample. It is also likely that the confinement of charges in a small layer may reduce the field in the depletion region or the layers may be permeable. 


\section{Conclusion}

The main significance of this work is the observations from the first and second scenarios which indicate that slight variations in layers separation can significantly impact the observed SH signal, this lead to a conclusion that in an experiment if the produced SH signal was measured to be very small it does not necessarily mean that induced second order nonlinearity is also small, and researchers should always keep the effect of layers spacing into consideration when studying multilayered structures. If the spacing in not consistent with coherence length then the measured SHG would be impacted and small.

As was observed in the second scenario the ratio of SHG increase almost as the square of the ratio of number of layers, the small discrepancy is because the model does not represent a continuous nonlinear medium. Our model suggests that our previous experiments could have produced even more SHG than was observed.

The smaller observed SHG could have been a consequence of confining charges within a small layer resulting in a weaker permanent electric field or perhaps the layers were permeable. 


\section{Chapter 7: Future Work}

The above mentioned questions such as the reason for the discrepancy between the experiment and model observations for generated SHG will be investigated further in experiments. To compare the findings of the model with experiments, new samples (with varying layer spacing) will need to be designed and measured. A study of layer permeability as a function of dopant concentration would also be of great interest. If the layers are permeable the charges at the interface would diffuse through the sample resulting in thinner and weaker depletion regions and a reduction of the SON. The occurrence of this phenomenon can be verified and by using a side-on SHG microscopy set-up to measure the non-linearity. Modeling this migration of charges between layers can be the next step in further developing the model to accurately simulate the real SHG profile in the multilayer structure (unlike the point sources we considered in our studies). 


\section{References}

[1] E. Udd and W. B. Spillman (Ed's), Fiber Optic Sensors: An Introduction for Engineers and Scientists (Wiley and Sons, Hoboken, NJ, 2011)

[2] R. Kashyap, Fiber Bragg Gratings (Academic Press, second ed., 2010).

[3] R. Boyd, Non-linear Optics (Academic Press, 2008)

[4] V. G. Dmitriev, G. G. Gurzadyan and D. N. Nikogosyan, Handbook of Nonlinear Optical Crystals (Springer-Verlag, 1991)

[5] U. Osterberg, W. Margulis, "Dye laser pumped by Nd:YAG laser pulses frequency doubled in a glass optical fiber", Optics Letters, 11 (8), pp. 516-518 (August 1986)

[6] R. A. Myers, N. Mukherjee, and S. R. J. Brueck, "Large second-order nonlinearity in poled fused silica”, Optics Letters, 16 (22), pp. 1732-1734 (November 1991)

[7] A. Okada, K. Ishii, K. Mito and K. Sasaki, "Phase-matched second-harmonic generation in novel corona poled glass waveguides," Applied Physics Letters, 60, pp. 2853-2855, (1992)

[8] P. Blazkiewicz, W. Xu, D. Wong, J. Canning, M. ${ }^{\circ}$ Asland, and G. Town, "Carbon dioxide laser-assisted poling of silicate-based optical fibers," Optics Letters, 25, pp. 200-202 (2000).

[9] T. Fujiwara, D. Wong, Y. Zhao, S. Fleming, S. Poole, and M. Sceats, "Electro-optic modulation in germanosilicate fibre with UV-excited poling," Electronics Letters, 31, pp. 573-575 (1995).

[10] C. Corbari, P. G. Kazansky, S. A. Slattery, and D. N. Nikogosyan, "Ultraviolet poling of pure fused silica by high-intensity femtosecond radiation,” Applied Physics Letters, 86, p. 071106 (2005).

[11] P. G. Kazansky, A. Kamal, and P. St. J. Russell, "High second-order nonlinearities induced in lead silicate glass by electron-beam irradiation," Optics Letters, 18, pp. 693-695 (1993).

[12] L. J. Henry, B. V. McGrath, T. G. Alley, and J. J. Kester, "Optical nonlinearity in fused silica by proton implantation," Journal of the Optical Society of America B, vol. 13, pp. 827-836, May 1996.

[13] A. C. Liu, M. J. F. Digonnet, and G. S. Kino, "Electro-optic phase modulation in a silica channel waveguide", Optics Letters, 19 (7), pp. 466-468 (April 1994)

[14] P. G. Kazansky, V. Pruneri, and P. St. J. Russel, "Blue-light generation by quasi-phasematched frequency doubling in thermally poled optical fibers" Optics Letters, 20 (8), pp. 843-845 (1995)

[15] Y. Quiquempois, N. Godbout, S. Lacroix, "Model of charge migration during thermal poling in silica glasses: Evidence of a voltage threshold for the onset of a second-order nonlinearity", Physical Review A, 66, pp. 043816-043817-14 (April 2002)

[16] R.H. Stolen and H. W. K. Tom, "Self-organized phase-matched harmonic generation in optical fibers", Optics Letters, 12 (8), pp. 585-587 (August 1987) 
[17] Eric Y. Zhu, Li Qian, L. G. Helt, Marco Liscidini, J. E. Sipe, Costantino Corbari, Albert Canagasabey, Morten Ibsen, and Peter G. Kazansky, "Measurement of $\chi 2$ symmetry in a poled fiber" Optics Letters, 35 (10), pp. 1530-1532 (May 2010)

[18] Huai-Yi Chen, Feng-Fan Chang, Jia-Cheng Liao, and Shiuh Chao, "Humidity effect on the decay of second-order nonlinearity in thermally poled fused silica" Optics Express, 14 (25), pp. 12334-12340

(December 2006)

[19] T. G. Alley, S.R.J. Brueck, and R. A. Myers" Space charge dynamics in thermally poled fused silica", Journal of Non-Crystalline Solids, 242, pp. 165-176 (July 1998)

[20] H. An and S. Fleming, "Time evolution of the second-order nonlinearity layer in thermally poled optical fiber”, Applied Physics Letters, 89, pp. 231105-231107 (2006).

[21] Arentoft, M. Kristensen, K. Pedersen, S. I. Bozhevolnyi, and P. Shi, "Poling of silica with silvercontaining electrodes," Electronics Letters, vol. 36, pp. 1635- 1636, September (2000)

[22] Luo, A. Biswas, A. Frauenglass and S. R. J. Brueck, "Large second-harmonic signal in thermally poled lead glass-silica waveguides," Applied Physics Letters, vol. 84, pp. 4935-4937, June (2004)

[23] D. Faccio, A. Busacca, D. W. J. Harwood, G. Bonfrate, V. Pruneri and P. G. Kazansky, "Effect of core-cladding interface on thermal poling of germanosilicate optical waveguides," Optics Communications, vol. 196, pp. 187-190, September (2001)

[24] A. Kudlinski, G. Martinelli, and Y. Quiquempois, "Influence of electrode composition on the secondorder nonlinearity profile in thermally poled silica glass", Optics Letters, 32 (13), pp. 1773-1775 (July 2007)

[25] Yadav, K., Smelser, C.W., Jacob, S., Blanchetiere, C., Callender, C.L., Albert, J., "Simultaneous corona poling of multiple glass layers for enhanced effective second-order optical nonlinearities", Applied Physics Letters, 99, 031109 (2011).

[26] Yadav K, Callender. C. L., Smelser C. W., Ledderhof C., Blanchetiere C., Jacob S., Albert J. "Giant enhancement of the second harmonic generation efficiency in poled multilayered silica structures", Optics Express, 19, 26975-26983 (2011).

[27] S. Kielich, "Doubling and mixing of laser light frequencies in crossed electric and magnetic fields", Optics communications, vol. 2, pp. 197-199, October (1970)

[28] Jesper Arentoft, Kjeld Pedersen, Sergey I. Bozhevolnyi, Martin Kristensen, Ping Yu, and Christian B. Nielsen. "Second-harmonic imaging of poled silica waveguides", Applied Physics Letters, vol. 76, No. 1, pp. 25-29. January 2000.

[29] Franken, P. A., Hill, A. E., “Generation of optical harmonics,” Phys. Rev. Lett., 7, 118 (1961)

[30] Miller, R. C., "Optical Second Harmonic Generation in piezoelectric crystals," Appl. Phys. Lett., 5, 17 (1964)

[31] Maker, P. D., Terhune, R. W., "Effects of dispersion and focusing on the production of optical harmonics," Phys. Rev. Lett., 8, 21 (1962)

[32] Jerphagnon, J., and Kurtz, S., "Optical nonlinear susceptibilities: Accurate relative values for Quartz, Ammonium dihydrogen phosphate and Potassium dihydrogen phosphate," Phys. Rev., B1, 1739 (1970)

[33] G. P. Agrawal, Nonlinear Fiber Optics, (Academic Press, Third ed. 2001)

[34] Zernike and Midwinter, Applied Nonlinear Optics, (Wiley, 1973) 
[35] H. An, S. Fleming, "Characterization of a second-order nonlinear layer profile in thermally poled optical fibers with second-harmonic microscopy", Optics Letters, Vol. 30, No. 8 (2005). 


\section{Appendix}

\section{The Matlab Model}

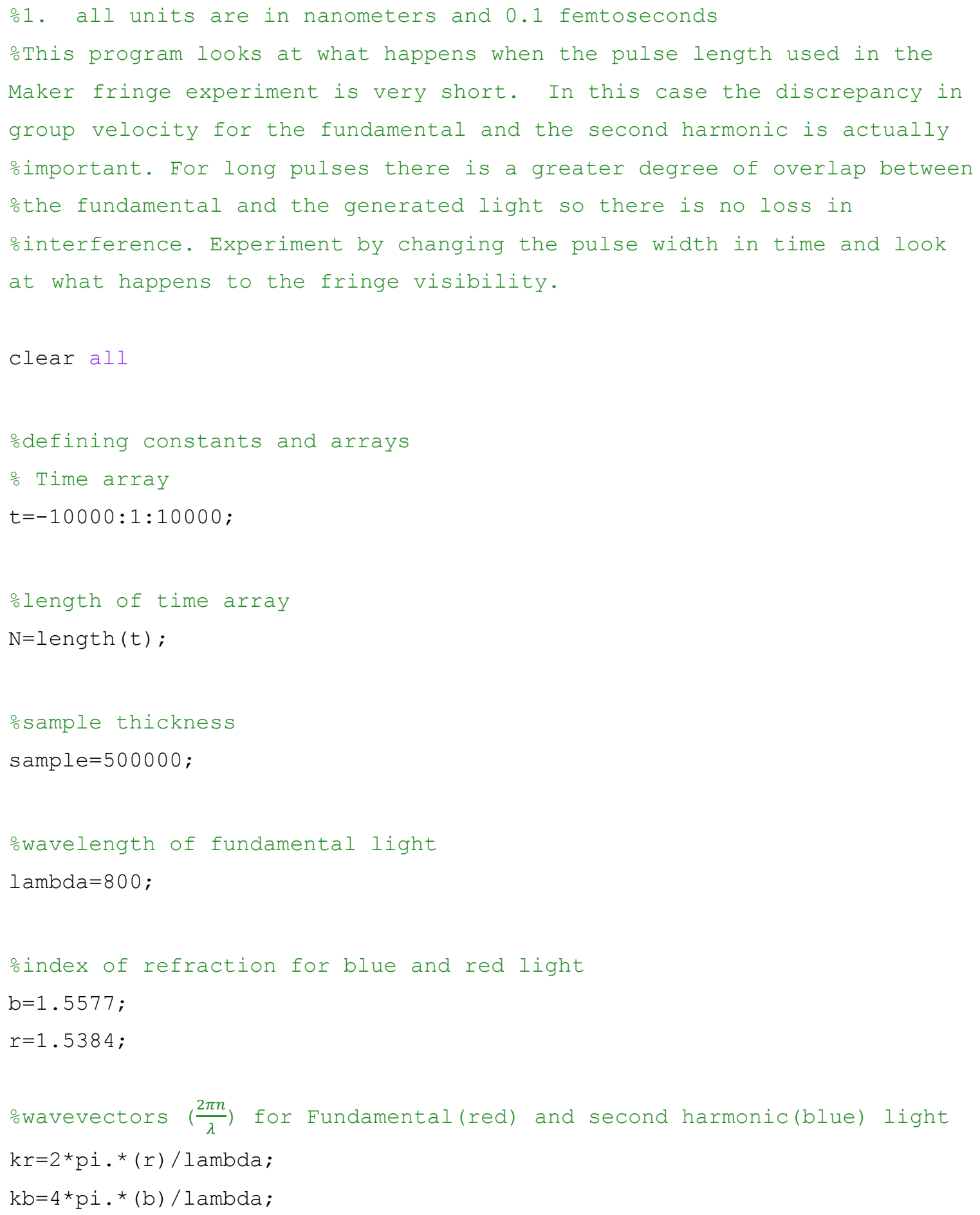




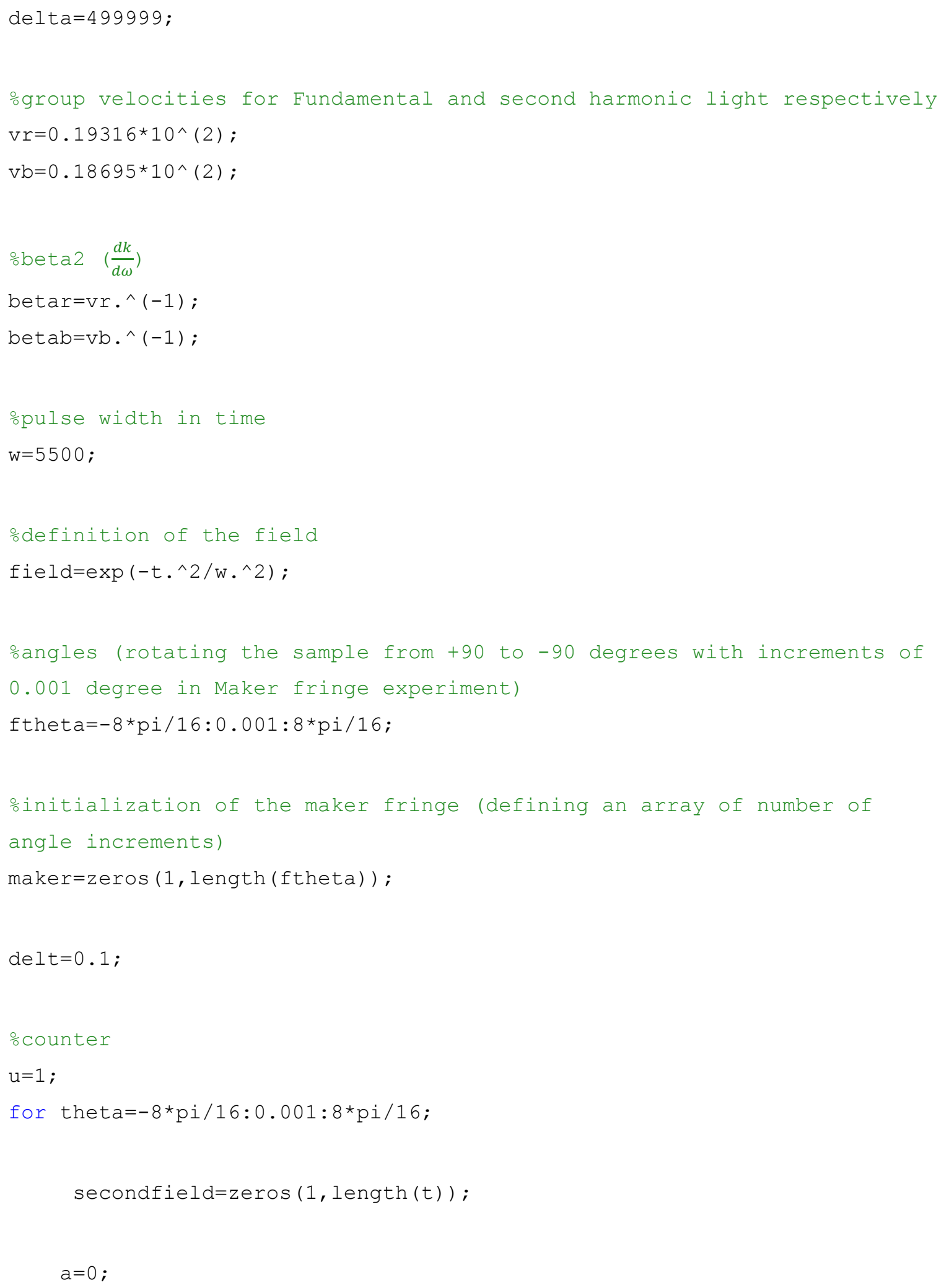




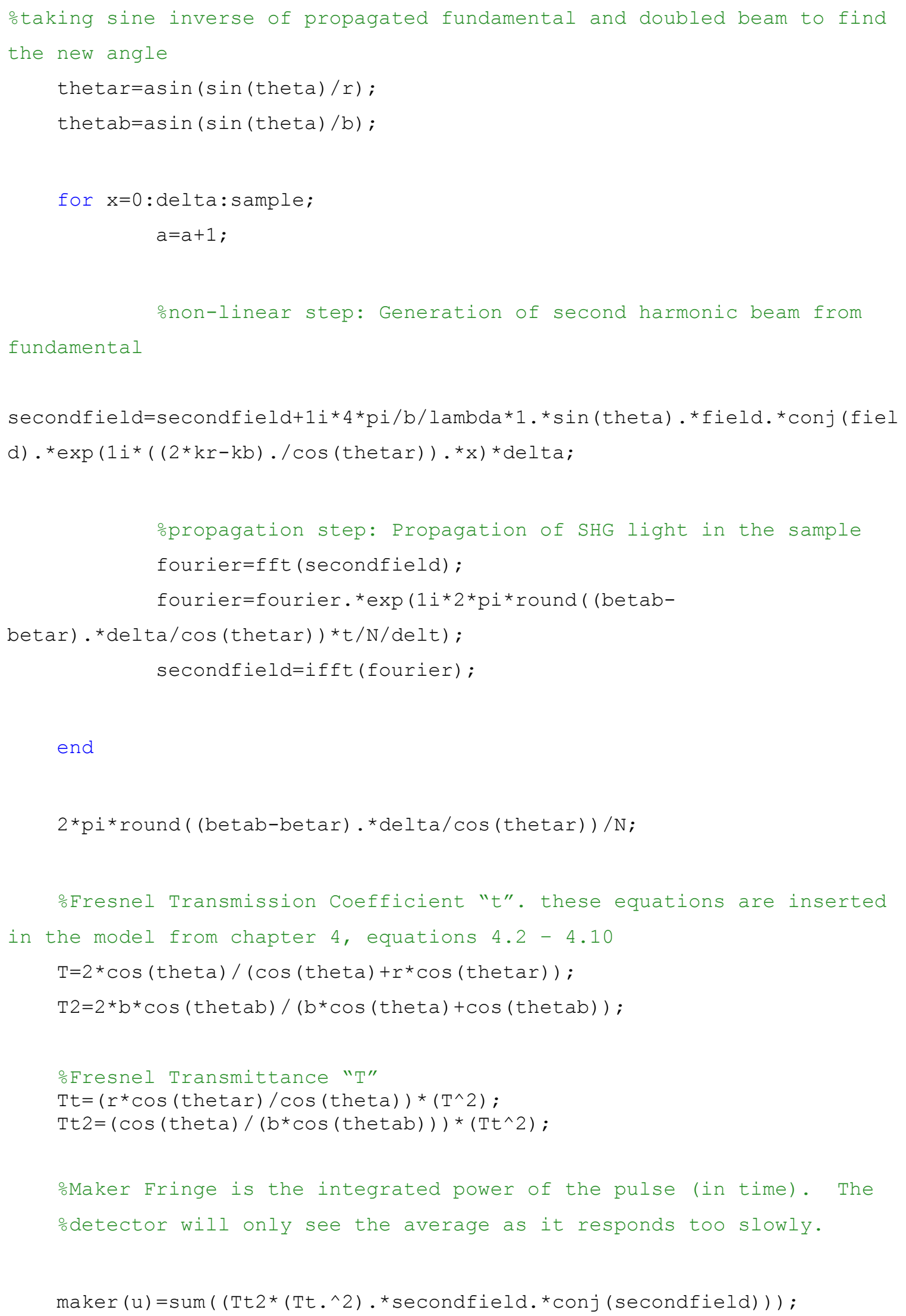


$\mathrm{u}=\mathrm{u}+1$ ；

end

maker=maker / (262.9);

figure, plot(ftheta*180/pi, maker) 